\title{
Efficient functional encryption for inner product with simulation-based security
}

\author{
Wenbo Liu ${ }^{1}$, Qiong Huang ${ }^{1,2^{*}}$ (D), Xinjian Chen ${ }^{1}$ and Hongbo Li ${ }^{1}$
}

\begin{abstract}
Functional encryption (FE) is a novel paradigm for encryption scheme which allows tremendous flexibility in accessing encrypted information. In FE, a user can learn specific function of encrypted messages by restricted functional key and reveal nothing else about the messages. Inner product encryption (IPE) is a special type of functional encryption where the decryption algorithm, given a ciphertext related to a vector $\boldsymbol{x}$ and a secret key related to a vector $\boldsymbol{y}$, computes the inner product $\boldsymbol{x} \cdot \boldsymbol{y}$. In this paper, we construct an efficient private-key functional encryption (FE) for inner product with simulation-based security, which is much stronger than indistinguishability-based security, under the External Decisional Linear assumption in the standard model. Compared with the existing schemes, our construction is faster in encryption and decryption, and the master secret key, secret keys and ciphertexts are shorter.
\end{abstract}

Keywords: Functional encryption, Inner product, Simulation-based security

\section{Introduction}

Traditional public key encryption provides all-or-nothing access to data, either recovering the entire plaintext or revealing nothing from the ciphertext. Functional Encryption (FE) (Dan et al. 2011; O'Neill 2010) is a very useful tool for non-interactive computation on encrypted data. In FE, the owner of master secret key msk can create a secret key $s k_{f}$ for a function $f$, which enables users to compute the value of $f(x)$ by decrypting a ciphertext of $x$ without revealing anything else about $x$. As cloud services are increasing rapidly, users' demand for computation on encrypted data is also increasing because cloud servers are by no means trustful. FE is one solution to this problem, providing a paradigm where users can compute a function $f$ on encrypted data using a secret key $s k_{f}$ without revealing anything else about the encrypted data using data to the cloud server.

One of the principal interests in FE is what class of functions $\mathcal{F}$ can be supported and what kind of security

*Correspondence: qhuang@scau.edu.cn

'College of Mathematics and Informatics, South China Agricultural University, 483 Wushan Road, 510642 Guangzhou, China

${ }^{2}$ Guangzhou Key Laboratory of Intelligent Agriculture, 483 Wushan Road, 510642 Guangzhou, China can be achieved. It started from identity-based encryption (Boneh and Franklin 2001), followed by attributed-based encryption (Goyal et al. 2006), hidden vector encryption (Iovino and Persiano 2008; Caro et al. 2012) and predicate encryption (Katz et al. 2008; Shen et al. 2009). Amazingly, recent works realize computation of general polynomial-size circuits (Garg et al. 2016), although they require expensive assumptions like indistinguishability obfuscation, which are far from being practical. Motivated by this unreality, (Abdalla et al. 2015) introduced a new non-generic FE scheme specialized for computation of the evaluation of inner product values, which is efficient and constructed from standard assumptions. As (Abdalla et al. 2015) mentioned in their work, innerproduct is a very useful tool for statistics because it can provide the weighted mean. In an inner product encryption (IPE) scheme, a ciphertext $c t_{x}$ is related to a vector $x \in \mathbb{Z}_{q}^{n}$ of length $n$ and a secret key $s k_{y}$ related to a vector $\boldsymbol{y} \in \mathbb{Z}_{q}^{n}$. Given the ciphertext and the secret key, the decryption algorithm computes the inner product $\boldsymbol{x} \cdot \boldsymbol{y}=$ $\sum_{i=1}^{n} x_{i} y_{i}$. Note that FE for inner-product is different from inner-product encryption (IPE) in the context of predicate encryption. In the phase of encryption and secret key generation of the inner product encryption (predicate 
encryption), a secret key corresponds to a predicate vector $y \in \mathbb{Z}_{q}^{n}$ and a ciphertext for a message $m$ comes along with an attribute vector $\boldsymbol{x} \in \mathbb{Z}_{q}^{n}$, the decryption algorithm outputs $m$ if $\boldsymbol{x} \cdot \boldsymbol{y}=0$. By contrast, the result of the IPE scheme in this paper is the actual value of the inner product.

Private-key IPE has several practical applications (Kim et al. 2018; Zhao et al. 2018) as well, such as biometric authentication and nearest-neighbor search on encrypted data. Biometric-based authentication system is prevalent. It is well-known that biometrics are inherently noisy, authentication should be successful when the supplied biometric is close to a user's credential stored in the system. It is achieved by computing the Hamming distance between them, which is the number of bits differing from each other. Private-key inner product encryption can be used to compute the Hamming distance between the bitstrings of two vectors. Another application of IPE is the nearest-neighbor search on encrypted data. Consider an encrypted database of files $F$. Given a file $f$, the problem of $k$-nearest neighbor search is to find the prior- $k$ files in $F$ that are the most similar to $f$. The common measure of file similarity is the Euclidean distance between the vector representations of files. Private-key IPE gives an efficient way of performing the nearest-neighbor search over an encrypted database. Readers could refer to (Kim et al. 2018; Zhao et al. 2018) for a detailed introduction to these applications of IPE.

\section{Related works}

The first construction of IPE was presented by Abdalla et al. (2015) who developed a selectively secure scheme under the indistinguishability-based security. Subsequently, (Agrawal et al. 2016) and (Abdalla et al. 2016) have designed adaptively secure IPE constructions where the messages $\boldsymbol{x}_{0}$ and $\boldsymbol{x}_{1}$ may be adaptively chosen in time, based on the previously collected information. However, these constructions are built with public key and do not support any forms of function privacy. Then, researchers explored the possibility of attaining function privacy in the context of IPE. Bishop et al. (2015) constructed a functionhiding IPE scheme in the private key domain under the well-studied Symmetric External Diffie-Hellman(SXDH) assumption, which satisfies an indistinguishability-based definition, and considered adaptive adversaries. Roughly speaking, an IPE scheme is function-hiding if the keys and ciphertexts reveal no additional information about both $\boldsymbol{x}$ and $\boldsymbol{y}$ beyond their inner product. However, their security model imposes a little unrealistic admissibility constraint on the adversary's queries. All ciphertexts queries $\boldsymbol{x}_{0}, \boldsymbol{x}_{1}$ and all secret key queries $\boldsymbol{y}_{0}, \boldsymbol{y}_{1}$ are restrained by $\boldsymbol{x}_{0} \cdot \boldsymbol{y}_{0}=$ $x_{0} \cdot y_{1}=x_{1} \cdot y_{0}=x_{1} \cdot y_{1}$. That makes the security of the scheme become weak. Datta et al. (2017) constructed a private-key function-hiding IPE scheme from the SXDH assumption that changed the restriction on adversaries' queries is only $\boldsymbol{x}_{0} \cdot \boldsymbol{y}_{0}=\boldsymbol{x}_{1} \cdot \boldsymbol{y}_{1}$. In their construction, secret keys and ciphertexts of $n$-dimensional vectors consist of $4 n+8$ groups elements. This was further improved upon in a work by Tomida et al. (2016) who gave a construction of a private-key function hiding IPE from the DLIN assumption where the secret keys and ciphertexts consist of $2 n+5$ groups elements. Recently, (Kim et al. 2019) put forth a construction of function-hiding IPE scheme with less parameter sizes and run time complexity than in Bishop et al. (2015); Datta et al. (2017). The scheme is proved simulation-based secure in the generic model of bilinear maps.

There are also several research works about Inner product encryption (Agrawal et al. 2015; Caro et al. 2013; Goldwasser et al. 2014; Abdalla et al. 2017); (Datta et al. 2018), such as Multi-Input inner product encryption(MIPE) and predicate encryption for inner product (Okamoto and Takashima 2009; Attrapadung and Libert 2010; Lewko et al. 2010; Okamoto 2011; Park 2011; Okamoto and Takashima 2012a; Okamoto and Takashima 2012b; Kawai and Takashima 2013; Zhenlin and Wei 2015; Zhang et al. 2019). In Goldwasser et al. (2014) introduced the definition of Multi-Input functional encryption, the functions can be evaluated on encrypted information to take multiple inputs, with each input corresponding to a different ciphertext. Abdalla et al. (2017) constructed the first scheme of Multi-Input inner product encryption which achieves message privacy, and (Datta et al. 2018) proposed a new scheme which they call unbounded private-key Multi-Input inner product functional encryption. Their scheme achieved function-hiding privacy, meanwhile they enabled the encryption of ciphertexts, and the generation of secret keys for unbounded vectors. In Dufour-Sans and Pointcheval (2019), described an unbounded inner product encryption which supported identity access control with succinct keys. Their construction is proven selectively secure in the random-oracle model based on the standard DBDH assumption. Tomida and Takashima (2018) did the similar research, but their construction didn't supported the function of identity access control. In Agrawal et al. (2020), resolved the question of simulation-based security for inner product encryption based on DDH, Paillier and LWE assumption. In 2008, the first predicate encryption for inner product was introduced by Katz et al. (2008), which allows evaluating predicates over $\mathbb{Z}_{N}$ using inner product, where $N$ is a composite number. In Okamoto and Takashima (2009) proposed the first hierarchical predicate encryption for inner product predicate, which allows a user with functionality that can delegate more restrictive functionality to another user, but their schemes had low inefficiency. Attrapadung and Libert (2010); Lewko et al. (2010); Okamoto (2011) constructed their IPE 
schemes respectively, which improves the efficiency of the previous scheme. However, the security proof of all previous studies based on non-standard assumptions. In order to address this issue, (Park 2011) proposed the first IPE scheme under the standard assumptions (i.e., decisional bilinear Diffie-Hellman (DBDH) and decisional linear (DLIN) assumptions). Okamoto and Takashima (2012a) proposed the first IPE scheme that is fully secure and fully attribute-hiding, and then (Okamoto and Takashima 2012b) further proposed the first unbounded IPE scheme that is also fully secure and fully attribute-hiding in the standard model under DLIN assumption. Kawai and Takashima (2013) introduced a new concept, called IPE with ciphertext conversion, which takes into account the security of predicate hiding. Zhenlin and Wei (2015) introduced another notion, called multi-party cloud computation IPE with multiplicative homomorphic property, which enables IPE to support multi-party cloud computation. Zhang et al. (2019) proposed a new IPE scheme based on double encryption system, which is proven to be adaptive security under weak attribute hiding model.

A lot of the problems mentioned above will lead an IPE scheme impractical and takes us the following problem:

Can we optimize the length of the master secret key, ciphertexts and secret keys with the simulation-based security?

\section{Our contribution}

We construct a more efficient and flexible private-key IPE scheme with simulation-based security. To ensure correctness, our scheme requires that the computation of inner products is within a polynomial range (Datta et al. 2016), where discrete logarithm of $g^{x \cdot y}$ can be found in polynomial time.

\section{Efficiency}

Our scheme is constructed based on dual paring vector spaces (DPVS). Namely, a master secret key is orthonormal bases of DPVS, secret keys and ciphertexts are vectors of DPVS, both key generation algorithm and encryption algorithm involve scalar multiplications on cyclic groups, and a decryption algorithm involves paring operations on bilinear paring groups. Our scheme is superior to other schemes, in terms of both necessary storage and computational efficiency.

\section{Assumption and flexibility}

The schemes of Bishop et al. (2015); Datta et al. (2016) are secure under the symmetric external Diffie-Hellman (SXDH) assumption, while our scheme is based on decision linear (DLIN) assumption or its variant (XDLIN). SXDH holds in only Type-3 bilinear pairing groups, DLIN and XDLIN hold in any type of bilinear pairing groups, so DLIN and XDLIN are weaker assumptions than SXDH.
For this reason, the schemes of Bishop et al. (2015) Datta et al. (2016) work in only Type-3 groups while we can use our scheme in any type of bilinear pairing groups.

\section{Security}

There are two notions of security for a FE scheme, indistinguishability-based security and simulation based security model. The former one requires that an adversary cannot distinguish between ciphertexts of any two messages $m_{0}, m_{1}$ with access to a secret key $s k_{f}$ of function $f$ such that $f\left(m_{0}\right)=f\left(m_{1}\right)$. By contrast, the latter one requires that the view of the adversary can be simulated by a simulator, given only access to secret keys and functions evaluated on the corresponding messages. Note that simulation-based security has higher security strength than indistinguishability-based security such that there exists an indistinguishability-based secure $\mathrm{FE}$ scheme for a certain functionality which is not able to be proved secure under simulation-based security. Our scheme achieves simulation-based security, which is more secure than indistinguishability-based security.

\section{Preliminary \\ Notation}

For a set $S, x \stackrel{\cup}{\leftarrow} S$ denotes that $x$ is uniformly chosen from $S$. For a probability distribution $X, x \stackrel{\mathrm{R}}{\leftarrow} X$ denotes that $x$ is chosen from $X$ according to its distributions. For a prime $q, \mathbb{Z}_{q}$ denotes a set of integers $\{0, \cdots, q-1\}$, and $\mathbb{Z}_{q}^{\times}$denotes a set of integers $\{1, \cdots, q-$ $1\}, 0$ denotes a zero vector. For an $n$-dimensional vector $\boldsymbol{x}, x_{i}(1 \leq i \leq n)$ denotes the $i$-th component of $\boldsymbol{x}$. For vectors $\boldsymbol{x}, \boldsymbol{y} \in \mathbb{Z}_{q}^{n}, \boldsymbol{x} \cdot \boldsymbol{y}$ denotes inner-product of $\boldsymbol{x}$ and $\boldsymbol{y}$ over $\mathbb{Z}_{q}$. For vector components, $0^{n}$ denotes a line of $n$ zeros, e.g., $\boldsymbol{a}:=(0,0,0,1)=\left(0^{3}, 1\right)$. Let $A=\left\{A_{n}\right\}_{n \in \mathbb{N}}$ and $B=\left\{B_{n}\right\}_{n \in \mathbb{N}}$ be distribution ensembles. We denote by $A \approx_{c} B$ that $A$ and $B$ are computationally indistinguishable. Let $n e g l(\lambda)$ be a negligible function in $\lambda . B^{T}$ denotes the transpose of matrix $\boldsymbol{B} . G L\left(n, \mathbb{Z}_{q}\right)$ denotes the general linear group of degree $n$ over $\mathbb{Z}_{q}$.

\section{Bilinear pairing groups}

Bilinear pairing groups are defined by the tuple $\left(q, \mathbb{G}_{1}, \mathbb{G}_{2}, \mathbb{G}_{T}, e\right)$, where $q$ is a prime, $\mathbb{G}_{1}, \mathbb{G}_{2}$ and $\mathbb{G}_{T}$ are cyclic groups of order $q$, and $e: \mathbb{G}_{1} \times \mathbb{G}_{2} \rightarrow \mathbb{G}_{T}$ is a map that has the following properties:

$$
\begin{aligned}
1 \text { Bilinearity: } \forall G_{1} \in \mathbb{G}_{1}, \forall G_{2} \in \mathbb{G}_{2}, \forall a, b \in \\
\\
\mathbb{Z}_{q}, e\left(a G_{1}, b G_{2}\right)=e\left(G_{1}, G_{2}\right)^{a b} . \\
2 \text { Non-degeneracy: if } \\
\forall G_{1} \in \mathbb{G}_{1}, G_{1} \neq 0, e\left(G_{1}, G_{2}\right)=1 \text {, then } G_{2}=0 .
\end{aligned}
$$

There are three types of bilinear groups according to whether efficient isomorphisms exist or not between $\mathbb{G}_{1}$ and $\mathbb{G}_{2}$. In Type-1 bilinear groups, both the isomorphism 
$\phi: \mathbb{G}_{2} \rightarrow \mathbb{G}_{1}$ and its inverse $\phi^{-1}: \mathbb{G}_{1} \rightarrow \mathbb{G}_{2}$ can be computed efficiently, i.e., $\mathbb{G}_{1}=\mathbb{G}_{2}$. In Type-2 bilinear groups, the isomorphism $\phi: \mathbb{G}_{2} \rightarrow \mathbb{G}_{1}$ is computed efficiently but its inverse is not. Type-3 groups do not have efficient isomorphisms between $\mathbb{G}_{1}$ and $\mathbb{G}_{2}$. Type- 1 groups are called symmetric bilinear pairing groups, and Type- 2 and Type3 are called asymmetric bilinear pairing groups. We use Type-3 groups to build our scheme in the paper. Let $\mathcal{G}_{\text {abpg }}$ be an asymmetric bilinear pairing group generators that takes $1^{\lambda}$ and outputs a description of $\left(q, \mathbb{G}_{1}, \mathbb{G}_{2}, \mathbb{G}_{T}, e\right)$ and generators of groups $G_{1} \neq 0 \in \mathbb{G}_{1}, G_{2} \neq 0 \in \mathbb{G}_{2}$. We denote the tuple $\left(q, \mathbb{G}_{1}, \mathbb{G}_{2}, \mathbb{G}_{T}, G_{1}, G_{2}, e\right)$ by param $\mathbb{G}_{\mathbb{G}}$.

\section{Dual pairing vector space}

Definition 1 (Lewko et al. 2010; Okamoto and Takashima 2009; Okamoto and Takashima 2015) (Dual Pairing Vector Spaces : DPVS): We briefly introduce the concept of DPVS. DPVS are defined by the tuple $\left(q, \mathbb{V}, \mathbb{V}^{*}, \mathbb{G}_{T}, \mathbb{A}, \mathbb{A}^{*}, \widetilde{e}\right)$, which is directly constructed from param $_{\mathbb{G}} \stackrel{\mathrm{R}}{\leftarrow} \mathcal{G}_{\text {abpg }}\left(1^{\lambda}\right) . \mathbb{V}:=\mathbb{G}_{1}^{n}$ and $\mathbb{V}^{*}:=\mathbb{G}_{2}^{n}$ are $n$-dimensional vector spaces, $\begin{aligned} & \mathbb{A}:=\left(\boldsymbol{a}_{1}, \cdots, \boldsymbol{a}_{n}\right)= {\left[\begin{array}{llll}G_{1} & 0 & \cdots & 0 \\ 0 & G_{1} & \cdots & 0 \\ \vdots & \vdots & \ddots & \vdots \\ 0 & 0 & \cdots & G_{1}\end{array}\right] \text { and } } \\ & \mathbb{A}^{*}:=\left(\boldsymbol{a}_{1}^{*}, \cdots, \boldsymbol{a}_{n}^{*}\right)=\left[\begin{array}{llll}G_{2} & 0 & \cdots & 0 \\ 0 & G_{2} & \cdots & 0 \\ \vdots & \vdots & \ddots & \vdots \\ 0 & 0 & \cdots & G_{2}\end{array}\right] \text { are }\end{aligned}$ canonical bases, where $\boldsymbol{a}_{i}:=\left(0^{i-1}, G_{1}, 0^{n-i}\right)$, $\boldsymbol{a}_{i}^{*}:=\left(0^{i-1}, G_{2}, 0^{n-i}\right)$, and $\tilde{e}: \mathbb{V} \times \mathbb{V}^{*} \rightarrow \mathbb{G}_{T}$ is $a$ pairing defined by $\tilde{e}(\boldsymbol{x}, \boldsymbol{y}):=\prod_{i=1}^{n} e\left(X_{i}, Y_{i}\right) \in \mathbb{G}_{T}$, where $\boldsymbol{x}:=\left(X_{1}, \cdots, X_{n}\right) \in \mathbb{V}, \boldsymbol{y}:=\left(Y_{1}, \cdots, Y_{n}\right) \in \mathbb{V}^{*}$.

Let $\left(q, \mathbb{V}, \mathbb{V}^{*}, \mathbb{G}_{T}, \mathbb{A}, \mathbb{A}^{*}, \widetilde{e}\right)$ be the output of algorithm $\mathcal{G}_{\text {dpvs }}\left(1^{\lambda}, n\right.$, param $\left._{\mathbb{G}}\right)$, where $n \in \mathbb{N}$. Then we describe random dual orthonormal bases as follows:

We randomly select a new non-singular matrix $X$ to do a linear transformation and achieve base change.

$$
\begin{gathered}
\psi \stackrel{U}{\leftarrow} \mathbb{Z}_{q}^{\times}, X:=\left(\chi_{i, j}\right)_{1 \leq i, j \leq n} \stackrel{U}{\leftarrow} G L\left(n, \mathbb{Z}_{q}\right),\left(v_{i, j}\right)_{1 \leq i, j \leq n} \\
:=\psi\left(\boldsymbol{X}^{T}\right)^{-1}, \\
\boldsymbol{b}_{i}:=\sum_{j=1}^{n} \chi_{i, j} \boldsymbol{a}_{j}=\left(\begin{array}{ll}
\boldsymbol{a}_{1} \cdots \boldsymbol{a}_{n}
\end{array}\right)\left(\begin{array}{c}
\chi_{i 1} \\
\vdots \\
\chi_{i n}
\end{array}\right), \\
\boldsymbol{b}_{i}^{*}:=\sum_{j=1}^{n} v_{i, j} \boldsymbol{a}_{j}^{*}=\left(\boldsymbol{a}_{1}^{*} \cdots \boldsymbol{a}_{n}^{*}\right)\left(\begin{array}{c}
v_{i 1} \\
\vdots \\
v_{i n}
\end{array}\right) \\
\quad \text { for } i=1, \cdots, n,
\end{gathered}
$$

$$
\begin{aligned}
\mathbb{B} & :=\left(\boldsymbol{b}_{1}, \cdots, \boldsymbol{b}_{n}\right), \\
\mathbb{B}^{*} & :=\left(\boldsymbol{b}_{1}^{*}, \cdots, \boldsymbol{b}_{n}^{*}\right), \\
g_{T} & :=e\left(G_{1}, G_{2}\right)^{\psi} .
\end{aligned}
$$

Let $\mathcal{G}_{\text {ob }}$ be the random dual orthonormal basis generator that takes $1^{\lambda}$ and a dimension of bases $n$ and outputs $\left(\right.$ param $\left._{\mathbb{G}}, \mathbb{B}, \mathbb{B}^{*}, g_{T}\right)$, where $\mathbb{B}, \mathbb{B}^{*}, g_{T}$ are computed as above. We denote the combination $\left(\right.$ param $\left._{\mathbb{G}}, g_{T}\right)$ by param $_{\mathbb{V}}$. For a vector $\boldsymbol{x}=\left(x_{1}, \cdots, x_{n}\right)^{T} \in \mathbb{Z}_{q}^{n}$ and a basis $\mathbb{B}:=\left(\boldsymbol{b}_{1}, \cdots, \boldsymbol{b}_{n}\right)$ we denote $\sum_{i=1}^{n} x_{i} \boldsymbol{b}_{i}=$

$$
\begin{aligned}
&\left(\begin{array}{lll}
x_{1} & \cdots & x_{n}
\end{array}\right)\left(\begin{array}{c}
\boldsymbol{b}_{1} \\
\vdots \\
\boldsymbol{b}_{n}
\end{array}\right) \operatorname{by}(\boldsymbol{x})_{\mathbb{B}} . \text { Then we have } \\
& \tilde{e}\left((\boldsymbol{x})_{\mathbb{A}},(\boldsymbol{y})_{\mathbb{A}^{*}}\right)=\prod_{i=1}^{n} e\left(x_{i} G_{1}, y_{i} G_{2}\right) \\
&=e\left(G_{1}, G_{2}\right)^{\sum_{i=1}^{n} x_{i} y_{i}}=e\left(G_{1}, G_{2}\right)^{\boldsymbol{x} \cdot \boldsymbol{y}},
\end{aligned}
$$

so

$$
\begin{aligned}
\tilde{e}\left((\boldsymbol{x})_{\mathbb{B}},(\boldsymbol{y})_{\mathbb{B}^{*}}\right) & =\tilde{e}\left((\boldsymbol{X x})_{\mathbb{A}},\left(\psi\left(X^{T}\right)^{-1} \boldsymbol{y}\right)_{\mathbb{A}^{*}}\right) \\
& =e\left(G_{1}, G_{2}\right)^{\psi \boldsymbol{X x} \cdot\left(X^{T}\right)^{-1} \boldsymbol{y}}=g_{T}^{\boldsymbol{x} \cdot \boldsymbol{y}} .
\end{aligned}
$$

\section{External decision linear assumption}

Definition 2 (Abe et al. 2016) We choose an arbitrary number $x \in\{1,2\}$. The XDLIN problem is to guess a bit $b \in\{0,1\}$, given $P_{b}$, where

$$
\begin{aligned}
& \operatorname{param}_{\mathbb{G}} \stackrel{R}{\leftarrow} \mathcal{G}_{\text {abpg }}\left(1^{\lambda}\right), \xi, \kappa, \delta, \sigma, \rho \stackrel{U}{\leftarrow} \mathbb{Z}_{q}, \\
& Y_{0}=(\delta+\sigma) G_{x}, Y_{1}=(\delta+\sigma+\rho) G_{x}, \\
& P_{b}=\left(\operatorname{param}_{\mathbb{G}}, \xi G_{1}, \kappa G_{1}, \delta \xi G_{1},\right. \\
&\left.\sigma \kappa G_{1}, \xi G_{2}, \kappa G_{2}, \delta \xi G_{2}, \sigma \kappa G_{2}, Y_{b}\right) .
\end{aligned}
$$

For any probabilistic polynomial time (PPT) adversary $\mathcal{A}$, if its advantage (defined as below) in solving XDLIN problem is negligible in $\lambda$, we say that the XDLIN assumption holds. Namely,

$$
\begin{aligned}
\operatorname{Adv}_{\mathcal{A}}{ }^{\mathrm{XDLIN}}(\lambda) & =\operatorname{Pr}\left[\mathcal{A}\left(1^{\lambda}, P_{0}\right) \rightarrow 1\right] \\
& -\operatorname{Pr}\left[\mathcal{A}\left(1^{\lambda}, P_{1}\right) \rightarrow 1\right] \leq \operatorname{negl}(\lambda) .
\end{aligned}
$$

\section{Private-key inner product encryption}

A private-key IPE scheme is composed of the following four PPT algorithms.

- IPE.Setup $\left(1^{\lambda}, n\right) \rightarrow(m s k, p p)$ : The setup algorithm takes as input the security parameters $1^{\lambda}$ and vector length $n$. Then it outputs a master secret key msk and public parameters $p p$.

- IPE.Encrypt $(m s k, p p, \boldsymbol{x}) \rightarrow c t_{\boldsymbol{x}}$ : The encryption algorithm takes as input the master secret key msk, 
the public parameters $p p$, and a vector $\boldsymbol{x} \in \mathbb{Z}_{q}^{n}$. Then it outputs a ciphertext $c t_{x}$.

- IPE.KeyGen $(m s k, p p, \boldsymbol{y}) \rightarrow s k_{y}$ : The key generation algorithm takes as input the master secret key msk, the public parameters $p p$, and a vector $\boldsymbol{y} \in \mathbb{Z}_{q}^{n}$. Then it outputs a secret key $s k_{y}$.

- IPE.Decryption $\left(p p, c t_{x}, s k_{y}\right) \rightarrow m \in \mathbb{Z}_{q}$ or $\perp$ : The decryption algorithm takes as input the public parameters pp, a ciphertext $c t_{x}$ and a secret key $s k_{y}$. Then it outputs either a value $m=\boldsymbol{x} \cdot \boldsymbol{y} \in \mathbb{Z}_{q}$ or a dedicated symbol $\perp$.

\section{Simulation-based security}

The simulation-based security (Caro et al. 2013; O'Neill 2010) in the Fig. 1 tries to capture the intuition that anything the adversary can compute from a ciphertext and the secret keys can be computed from the secret keys and the values of the corresponding functions on the underlying message. For an IPE scheme, if there exists a PPT adversary $\mathcal{A}=\left(\mathcal{A}_{1}, \mathcal{A}_{2}\right)$ and a PPT simulator $\mathcal{S}$, we define two experiments $\operatorname{REAL}_{\mathcal{A}}\left(1^{\lambda}\right), \operatorname{IDEAL}_{\mathcal{A}, \mathcal{S}}\left(1^{\lambda}\right)$ in the box, let $q_{1}$ be the number of challenge messages output by $\mathcal{A}_{1}$ and $q_{2}$ be the number of secret key queries in the first stage. The oracle $\mathcal{O}$ and $\mathcal{O}^{\prime}$ are defined as follows:

1 The oracle $\mathcal{O}(m s k, \cdot)=$ IPE.KeyGen $(m s k, \cdot, \cdot)$.

2 The oracle $\mathcal{O}^{\prime}(m s k, s t, \cdot)$ is the second stage of the simulator, namely algorithm $\mathcal{S}^{\left\{\boldsymbol{x}^{(l)} \cdot \boldsymbol{y}^{(\mu)}\right\}}{ }_{(m s k, s t, \cdot) \text {, for }}$ $l \in\left\{1, \cdots, q_{1}\right\}, \mu \in\left\{1, \cdots, q_{2}\right\}$, where $\boldsymbol{x}^{(l)}$ and $\boldsymbol{y}^{(\mu)}$ are inputs of the $l$-th ciphertext query and the $\mu$-th secret key query by $\mathcal{A}_{1}$, respectively. Note that the simulator algorithm $\mathcal{S}$ is stateful so that after each invocation, it updates the state st which is carried over to its next invocation.
An IPE scheme is simulation-based secure if there exists a PPT simulator $\mathcal{S}$ such that, for all PPT adversaries $\mathcal{A}$, the outputs of the following two experiments are computationally indistinguishable:

$$
\operatorname{REAL}_{\mathcal{A}}\left(1^{\lambda}\right) \approx_{c} \operatorname{IDEAL}_{\mathcal{A}, \mathcal{S}}\left(1^{\lambda}\right) .
$$

\section{Preliminary problems of security proof}

In this section, we will introduce six lemmas and their security proofs. we firstly consider the following problems and use them to prove the security of our scheme.

Definition 3 Problem 1 is to guess $b \in\{0,1\}$, given $\left(\right.$ param $\left._{\mathbb{V}}, \mathbb{B}, \widehat{\mathbb{B}}^{*}, \boldsymbol{y}_{b}, \kappa G_{1}, \xi G_{2}\right)$, where

$$
\begin{aligned}
& \operatorname{param}_{\mathbb{G}} \stackrel{\mathbb{R}}{\leftarrow} \mathcal{G}_{\text {abpg }}\left(1^{\lambda}\right), \\
& X:=\left(\chi_{i, j}\right)_{1 \leq i, j \leq 3} \stackrel{\cup}{\leftarrow} G L\left(3, \mathbb{Z}_{q}\right),\left(v_{i, j}\right)_{1 \leq i, j \leq 3} \\
& :=\left(X^{T}\right)^{-1}, \\
& \kappa, \xi \stackrel{\cup}{\leftarrow} \mathbb{Z}_{q}^{\times}, \boldsymbol{b}_{i}:=\kappa \sum_{j=1}^{3} \chi_{i, j} \boldsymbol{a}_{j}, \boldsymbol{b}_{i}^{*} \\
& :=\xi \sum_{j=1}^{3} v_{i, j} \boldsymbol{a}_{j}^{*} \text { for } i=1,2,3, \\
& \mathbb{B}:=\left(\boldsymbol{b}_{1}, \boldsymbol{b}_{2}, \boldsymbol{b}_{3}\right), \widehat{\mathbb{B}}^{*}:=\left(\boldsymbol{b}_{1}^{*}, \boldsymbol{b}_{3}^{*}\right), \\
& g_{T}:=e\left(G_{1}, G_{2}\right)^{\kappa \xi}, \\
& \delta, \sigma \stackrel{\cup}{\leftarrow} \mathbb{Z}_{q}, \rho \stackrel{\cup}{\leftarrow} \mathbb{Z}_{q}^{\times}, \boldsymbol{y}_{0}=(\delta, 0, \sigma)_{\mathbb{B}} \text { and } \\
& \boldsymbol{y}_{1}=(\delta, \rho, \sigma)_{\mathbb{B}} .
\end{aligned}
$$

$$
\begin{aligned}
& \text { Experiment } \operatorname{REAL}_{\mathcal{A}}\left(1^{\lambda}\right) \text { : } \\
& (m s k, p p) \stackrel{\$}{\leftarrow} \mathrm{IPE} . \operatorname{Setup}\left(1^{\lambda}, n\right) \\
& (\boldsymbol{x}, s t) \stackrel{\$}{\leftarrow} \mathcal{A}_{1}^{\mathrm{IPE} . \operatorname{KeyGen}(m s k, \cdot, \cdot)}(p p) \\
& c t_{\boldsymbol{x}^{(l)}} \stackrel{\$}{\leftarrow} \text { IPE.Encrypt }(m s k, p p, \boldsymbol{x}) \\
& \text { for } l \in\left\{1, \cdots, q_{1}\right\} \\
& \alpha \stackrel{\$}{\leftarrow} \mathcal{A}_{2} \mathcal{O}(m s k, \cdot, \cdot)\left(\left\{c t_{\boldsymbol{x}(l)}\right\}_{l \in\left\{1, \cdots, q_{1}\right\}}, s t\right) \\
& \text { Experiment } \operatorname{IDEAL}_{\mathcal{A}, \mathcal{S}}\left(1^{\lambda}\right) \text { : } \\
& (m s k, p p) \stackrel{\$}{\leftarrow} \text { IPE.Setup }\left(1^{\lambda}, n\right) \\
& (\boldsymbol{x}, s t) \stackrel{\$}{\leftarrow} \mathcal{A}_{1}{ }^{\mathrm{IPE} .} \operatorname{KeyGen}(m s k, \cdot, \cdot)(p p) \\
& \left(\left\{c t_{\boldsymbol{x}^{(l)}}\right\}_{l \in\left\{1, \cdots, q_{1}\right\}}\right) \stackrel{\$}{\leftarrow} \mathcal{S}_{1}\left(\left\{\boldsymbol{x}^{(l)} \cdot \boldsymbol{y}^{(\mu)}\right\}_{l \in\left\{1, \cdots, q_{1}\right\}, \mu \in\left\{1, \cdots, q_{2}\right\}}\right) \\
& \text { where } \boldsymbol{y}^{(1)}, \cdots, \boldsymbol{y}^{\left(q_{2}\right)} \text { are key queries made by } \mathcal{A}_{1} \\
& \alpha \stackrel{\$}{\leftarrow} \mathcal{A}_{2} \mathcal{O}^{\prime}(m s k, s t, \cdot)\left(\left\{c t{ }_{\boldsymbol{x}}^{(l)}\right\}_{l \in\left\{1, \cdots, q_{1}\right\}}, s t\right)
\end{aligned}
$$

Fig. 1 Simulation-based security 
Definition 4 Problem $1^{\prime}$ is to guess $b \in\{0,1\}$, given $\left(\right.$ param $\left._{\mathbb{V}}, \mathbb{B}^{*}, \widehat{\mathbb{B}}, \boldsymbol{y}_{b}^{*}, \kappa G_{1}, \xi G_{2}\right)$, where

$$
\begin{aligned}
& \operatorname{param}_{\mathbb{G}} \stackrel{R}{\leftarrow} \mathcal{G}_{\text {abpg }}\left(1^{\lambda}\right), \\
& \boldsymbol{X}:=\left(\chi_{i, j}\right)_{1 \leq i, j \leq 3} \stackrel{\cup}{\leftarrow} G L\left(3, \mathbb{Z}_{q}\right),\left(v_{i, j}\right)_{1 \leq i, j \leq 3} \\
& :=\left(X^{T}\right)^{-1} \text {, } \\
& \kappa, \xi \stackrel{\cup}{\leftarrow} \mathbb{Z}_{q}^{\times}, \boldsymbol{b}_{i}:=\kappa \sum_{j=1}^{3} \chi_{i, j} \boldsymbol{a}_{j}, \boldsymbol{b}_{i}^{*} \\
& :=\xi \sum_{j=1}^{3} v_{i, j} \boldsymbol{a}_{j}^{*} \text { for } i=1,2,3, \\
& \mathbb{B}^{*}:=\left(\boldsymbol{b}_{1}^{*}, \boldsymbol{b}_{2}^{*}, \boldsymbol{b}_{3}^{*}\right), \widehat{\mathbb{B}}:=\left(\boldsymbol{b}_{1}, \boldsymbol{b}_{3}\right), \\
& g_{T}:=e\left(G_{1}, G_{2}\right)^{\kappa \xi} \text {, } \\
& \delta, \sigma \stackrel{\cup}{\leftarrow} \mathbb{Z}_{q}, \rho \stackrel{\cup}{\leftarrow} \mathbb{Z}_{q}^{\times}, \boldsymbol{y}_{0}^{*}=(\delta, 0, \sigma)_{\mathbb{B}^{*}} \text { and } \boldsymbol{y}_{1}^{*} \\
& =(\delta, \rho, \sigma)_{\mathbb{B}^{*}} .
\end{aligned}
$$

Definition 5 Problem 2 is to guess $a$ bit $b \in\{0,1\}$, given $\left(\right.$ param $\left._{\mathbb{V}}, \widehat{\mathbb{B}}, \widehat{\mathbb{B}}^{*}, \boldsymbol{g}_{b}\right)$, where

$$
\begin{aligned}
& \left(\mathbb{B}, \mathbb{B}^{*}, \operatorname{param}_{\mathbb{V}}\right) \stackrel{\cup}{\leftarrow} \mathcal{G}_{\mathrm{ob}}\left(1^{\lambda}, n+5\right), \\
& \widehat{\mathbb{B}}=\left\{\boldsymbol{b}_{1}, \cdots, \boldsymbol{b}_{n}, \boldsymbol{b}_{n+1}, \boldsymbol{b}_{n+3}\right\}, \\
& \widehat{\mathbb{B}}^{*}=\left\{\boldsymbol{b}_{1}^{*}, \cdots, \boldsymbol{b}_{n}^{*}, \boldsymbol{b}_{n+2}^{*}, \boldsymbol{b}_{n+4}^{*}\right\}, \\
& \alpha, \eta \stackrel{\cup}{\leftarrow} \mathbb{Z}_{q}, \gamma^{\prime} \stackrel{\cup}{\leftarrow} \mathbb{Z}_{q}^{\times}, \\
& \boldsymbol{g}_{0}=\left(0^{n}, \alpha, 0, \eta, 0,0\right)_{\mathbb{B}} \text { and } \boldsymbol{g}_{1}=\left(0^{n}, \alpha, 0, \eta, 0, \gamma^{\prime}\right)_{\mathbb{B}} .
\end{aligned}
$$

Definition 6 Problem3 is to guess a bit $b \in\{0,1\}$, given $\left(\right.$ param $\left._{\mathbb{V}}, \widehat{\mathbb{B}}, \widehat{\mathbb{B}}^{*}, \boldsymbol{g}_{b}\right)$, where

$$
\begin{aligned}
& \left(\mathbb{B}, \mathbb{B}^{*}, \operatorname{param}_{\mathbb{V}}\right) \stackrel{\mathrm{R}}{\leftarrow} \mathcal{G}_{\mathrm{ob}}\left(1^{\lambda}, n+5\right), \\
& \widehat{\mathbb{B}}=\left\{\boldsymbol{b}_{1}, \cdots, \boldsymbol{b}_{n}, \boldsymbol{b}_{n+1}, \boldsymbol{b}_{n+5}\right\}, \\
& \widehat{\mathbb{B}}^{*}=\left\{\boldsymbol{b}_{1}^{*}, \cdots, \boldsymbol{b}_{n}^{*}, \boldsymbol{b}_{n+2}^{*}, \boldsymbol{b}_{n+4}^{*}\right\}, \\
& \alpha, \eta \stackrel{\cup}{\leftarrow} \mathbb{Z}_{q}, \gamma^{\prime} \stackrel{\cup}{\leftarrow} \mathbb{Z}_{q}^{\times}, \\
& \boldsymbol{g}_{0}=\left(0^{n}, \alpha, 0, \eta, 0, \gamma^{\prime}\right) \text { and } \boldsymbol{g}_{1}=\left(0^{n}, \alpha, 0,0,0, \gamma^{\prime}\right)_{\mathbb{B}} .
\end{aligned}
$$

Definition 7 Problem4 is to guess a bit $b \in\{0,1\}$, given $\left(\right.$ param $\left._{\mathbb{V}}, \widehat{\mathbb{B}}, \widehat{\mathbb{B}}^{*}, \boldsymbol{g}_{b}^{*}\right)$, where

$$
\begin{aligned}
& \left(\mathbb{B}, \mathbb{B}^{*}, \operatorname{param}_{\mathbb{V}}\right) \stackrel{R}{\leftarrow} \mathcal{G}_{\mathrm{ob}}\left(1^{\lambda}, n+5\right), \\
& \widehat{\mathbb{B}}=\left\{\boldsymbol{b}_{1}, \cdots, \boldsymbol{b}_{n}, \boldsymbol{b}_{n+1}, \boldsymbol{b}_{n+5}\right\}, \\
& \widehat{\mathbb{B}}^{*}=\left\{\boldsymbol{b}_{1}^{*}, \cdots, \boldsymbol{b}_{n}^{*}, \boldsymbol{b}_{n+2}^{*}, \boldsymbol{b}_{n+4}^{*}\right\}, \\
& \beta, \theta \stackrel{\cup}{\leftarrow} \mathbb{Z}_{q}, \tau^{\prime} \cup \mathbb{Z}_{q}^{\times}, \\
& \boldsymbol{g}_{0}^{*}=\left(0^{n}, 0, \beta, 0, \theta, 0\right)_{\mathbb{B}^{*}} \text { and } \boldsymbol{g}_{1}^{*}=\left(0^{n}, 0, \beta, \tau^{\prime}, \theta, 0\right)_{\mathbb{B}^{*}} .
\end{aligned}
$$

Definition 8 Problem5 is to guess a bit $b \in\{0,1\}$, given $\left(\right.$ param $\left._{\mathbb{V}}, \widehat{\mathbb{B}}, \widehat{\mathbb{B}}^{*}, \boldsymbol{g}_{b}^{*}\right)$, where

$$
\begin{aligned}
& \left(\mathbb{B}, \mathbb{B}^{*}, \operatorname{param}_{\mathbb{V}}\right) \stackrel{\mathbb{R}}{\leftarrow} \mathcal{G}_{\mathrm{ob}}\left(1^{\lambda}, n+5\right), \\
& \widehat{\mathbb{B}}:=\left(\boldsymbol{b}_{1}, \cdots, \boldsymbol{b}_{n}, \boldsymbol{b}_{n+1}, \boldsymbol{b}_{n+5}\right), \\
& \widehat{\mathbb{B}}^{*}:=\left\{\boldsymbol{b}_{1}^{*}, \cdots, \boldsymbol{b}_{n}^{*}, \boldsymbol{b}_{n+2}^{*}, \boldsymbol{b}_{n+3}^{*}\right\}, \\
& \beta, \theta \stackrel{\cup}{\leftarrow} \mathbb{Z}_{q}, \tau^{\prime} \stackrel{\cup}{\leftarrow} \mathbb{Z}_{q}^{\times}, \\
& \boldsymbol{g}_{0}^{*}=\left(0^{n}, 0, \beta, \tau^{\prime}, \theta, 0\right)_{\mathbb{B}^{*}} \text { and } \boldsymbol{g}_{1}^{*}=\left(0^{n}, 0, \beta, \tau^{\prime}, 0,0\right)_{\mathbb{B}^{*}} .
\end{aligned}
$$

For a PPT algorithm $\mathcal{A}$, the advantage of $\mathcal{A}$ against Problem $n\left(n=1,1^{\prime}, 2,3,4,5\right)$ is defined as

$$
\begin{aligned}
\operatorname{Adv}_{\mathcal{A}}^{\mathrm{Pn}}(\lambda) & :=\mid \operatorname{Pr}\left[\mathcal{A}\left(1^{\lambda}, P_{0}\right) \rightarrow 1\right] \\
& -\operatorname{Pr}\left[\mathcal{A}\left(1^{\lambda}, P_{1}\right) \rightarrow 1\right] \mid,
\end{aligned}
$$

where $P_{b}$ is an instance of Problem $n$ defined above. Then following six lemmas hold.

Lemma 1 For all PPT adversary $\mathcal{B}$ for Problem1, there exists a PPT adversary $\mathcal{A}$ such that $\operatorname{Adv}_{\mathcal{B}}^{\mathrm{P} 1}(\lambda) \leq$ $\operatorname{Adv}_{\mathcal{A}}^{\mathrm{XDLIN}}(\lambda)+5 / q$.

Proof We construct a PPT adversary $\mathcal{A}$ for the XDLIN problem from any PPT adversary $\mathcal{B}$ for Problem $1 . \mathcal{A}$ is given an instance of XDLIN problem and sets

$$
\begin{aligned}
& g_{T}:=e\left(\kappa G_{1}, \xi G_{2}\right), \text { param }_{\mathrm{P} 1}:=\left(\operatorname{param}_{\mathbb{G}}, g_{T}\right), \\
& \boldsymbol{u}_{1}:=(\xi, 0,1)_{\mathbb{A}}=\left(\xi G_{1}, 0, G_{1}\right), \\
& \boldsymbol{u}_{2}:=(0,0,1)_{\mathbb{A}}=\left(0,0, G_{1}\right), \\
& \boldsymbol{u}_{3}:=(0, \kappa, 1)_{\mathbb{A}}=\left(0, \kappa G_{1}, G_{1}\right), \\
& \boldsymbol{u}_{1}^{*}:=(\kappa, 0,0)_{\mathbb{A}^{*}}=\left(\kappa G_{2}, 0,0\right), \\
& \boldsymbol{u}_{2}^{*}:=(-\kappa,-\xi, \kappa \xi)_{\mathbb{A}^{*}}=\left(-\kappa G_{2},-\xi G_{2}, \kappa \xi G_{2}\right), \\
& \boldsymbol{u}_{3}^{*}:=(0, \xi, 0)_{\mathbb{A}^{*}}=\left(0, \xi G_{2}, 0\right), \\
& \boldsymbol{w}_{b}:=\left(\delta \xi G_{1}, \sigma \kappa G_{1}, Y_{b}\right) .
\end{aligned}
$$

$\mathcal{A}$ can compute $\boldsymbol{u}_{1}, \boldsymbol{u}_{2}, \boldsymbol{u}_{3}, \boldsymbol{u}_{1}^{*}, \boldsymbol{u}_{3}^{*}$. Then it generates a random linear transformation $W$ on $\mathbb{G}^{3}$ to get a new group of bases and sets

$$
\begin{aligned}
& \boldsymbol{b}_{i}:=W\left(\boldsymbol{u}_{i}\right) \text { for } i=1,2,3 \\
& \boldsymbol{b}_{i}^{*}:=\left(W^{-1}\right)^{T}\left(\boldsymbol{u}_{i}^{*}\right) \text { for } i=1,3, \\
& \mathbb{B}:=\left(\boldsymbol{b}_{1}, \boldsymbol{b}_{2}, \boldsymbol{b}_{3}\right), \widehat{\mathbb{B}}^{*}:=\left(\boldsymbol{b}_{1}^{*}, \boldsymbol{b}_{3}^{*}\right), \boldsymbol{y}_{b}:=W\left(\boldsymbol{w}_{b}\right) .
\end{aligned}
$$

Then $\mathcal{A}$ gives $\left(\right.$ param $\left._{P 1}, \mathbb{B}, \widehat{\mathbb{B}}^{*}, \boldsymbol{y}_{b}, \kappa G_{1}, \xi G_{2}\right)$ to $\mathcal{B}$, and outputs $b^{\prime}$ if $\mathcal{B}$ outputs $b^{\prime}$,

If $b=0$ and $Y_{b}=Y_{0}=(\delta+\sigma) G_{1}$, then $\boldsymbol{w}_{0}=$ $\left(\delta \xi G_{1}, \sigma \kappa G_{1},(\delta+\sigma) G_{1}\right)=(\delta \xi, \sigma \kappa,(\delta+\sigma))_{\mathbb{A}}=\delta \boldsymbol{u}_{1}+$ $\sigma \boldsymbol{u}_{2}$ and $\boldsymbol{y}_{0}=W\left(\boldsymbol{w}_{0}\right)=W\left(\delta \boldsymbol{u}_{1}+\sigma \boldsymbol{u}_{2}\right)=(\delta, 0, \sigma)_{\mathbb{B}}$, when $\kappa, \xi \neq 0$, with probability $2 / q$.

If $b=1$ and $Y_{b}=Y_{1}=(\delta+\rho+$ $\sigma) G_{1}$, then $\boldsymbol{w}_{1}=\left(\delta \xi G_{1}, \sigma \kappa G_{1},(\delta+\rho+\sigma) G_{1}\right)=$ $(\delta \xi, \sigma \kappa,(\delta+\sigma+\rho))_{\mathbb{A}}=\delta \boldsymbol{u}_{1}+\rho \boldsymbol{u}_{2}+\sigma \boldsymbol{u}_{3}$ and $\boldsymbol{y}_{1}=$ 
$W\left(\boldsymbol{w}_{1}\right)=W\left(\delta \boldsymbol{u}_{1}+\rho \boldsymbol{u}_{2}+\sigma \boldsymbol{u}_{3}\right)=(\delta, \rho, \sigma)_{\mathbb{B}}$, when $\kappa, \xi, \rho \neq 0$, except with probability $3 / q$.

It is the same as an instance of Problem 1 . If $\mathcal{B}$ succeeds in solving Problem1, so does $\mathcal{A}$ in solving XDLIN porblem.

Lemma 2 For all PPT adversary $\mathcal{B}$ for Problem1', there exists a PPT adversary $\mathcal{A}$ such that $\operatorname{Adv}_{\mathcal{B}}^{\mathrm{P} 1^{\prime}}(\lambda) \leq$ $\operatorname{Adv}_{\mathcal{A}}^{\mathrm{XDLIN}}(\lambda)+5 / q$.

Proof The proof follows in the same condition as Lemma 1.

Lemma 3 For all PPT adversary $\mathcal{B}$ for Problem2, there exists a PPT adversary $\mathcal{A}$ such that $\operatorname{Adv}_{\mathcal{B}}^{\mathrm{P} 2}(\lambda) \leq \operatorname{Adv}_{\mathcal{A}}^{\mathrm{P} 1}(\lambda)$.

Proof We construct a PPT adversary $\mathcal{A}$ for Problem 1 from any PPT adversary $\mathcal{B}$ for Problem2. $\mathcal{A}$ is given an instance of Problem $1\left(\right.$ param $\left._{\mathrm{P} 1}, \mathbb{B}, \widehat{\mathbb{B}}^{*}, \boldsymbol{y}_{b}, \kappa G_{1}, \xi G_{2}\right)$. Then $\mathcal{A}$ generates a random linear transformation $W$ on $\mathbb{G}^{n+5}$, and sets

$$
\begin{aligned}
\operatorname{param}_{\mathbb{V}}: & =\operatorname{param}_{\mathrm{P} 1} \\
\boldsymbol{d}_{i}: & =W\left(0^{i+2}, \kappa G_{1}, 0^{n+2-i}\right) \text { for } i=1, \cdots, n \\
\boldsymbol{d}_{n+1}: & =W\left(\boldsymbol{b}_{1}, 0^{n+2}\right), \boldsymbol{d}_{n+2}:=W\left(0^{n+2}, \kappa G_{1}, 0^{2}\right), \\
\boldsymbol{d}_{n+3}: & =W\left(\boldsymbol{b}_{3}, 0^{n+2}\right), \boldsymbol{d}_{n+4}:=W\left(0^{n+4}, \kappa G_{1}\right), \\
\boldsymbol{d}_{n+5}: & : W\left(\boldsymbol{b}_{2}, 0^{n+2}\right), \\
\boldsymbol{d}_{i}^{*}: & \left((W)^{-1}\right)^{T}\left(0^{i+2}, \xi G_{2}, 0^{n+2-i}\right) \\
& \quad \text { for } i=1, \cdots, n \\
\boldsymbol{d}_{n+1}^{*}: & \left((W)^{-1}\right)^{T}\left(\boldsymbol{b}_{1}^{*}, 0^{n+2}\right), \\
\boldsymbol{d}_{n+2}^{*}: & =W\left(0^{n+2}, \xi G_{2}, 0^{2}\right), \\
\boldsymbol{d}_{n+3}^{*}: & \left((W)^{-1}\right)^{T}\left(\boldsymbol{b}_{3}^{*}, 0^{n+2}\right), \\
\boldsymbol{d}_{n+4}^{*}: & \left((W)^{-1}\right)^{T}\left(0^{n+4}, \xi G_{2}\right), \\
\boldsymbol{d}_{n+5}^{*}: & =W\left(\boldsymbol{b}_{2}^{*}, 0^{n+2}\right), \\
\boldsymbol{h}_{b} & =W\left(\boldsymbol{y}_{b}, 0^{n+2}\right) \\
\mathbb{D}: & \left(\boldsymbol{d}_{1}, \cdots, \boldsymbol{d}_{n+5}\right), \mathbb{D}^{*}:=\left(\boldsymbol{d}_{1}^{*}, \cdots, \boldsymbol{d}_{n+5}^{*}\right)
\end{aligned}
$$

We can see that $\left(\mathbb{D}, \mathbb{D}^{*}\right)$ are dual orthonormal bases. $\mathcal{A}$ does not have $\boldsymbol{b}_{2}^{*}$ but it can compute

$$
\begin{aligned}
\widehat{\mathbb{D}} & :=\left(\boldsymbol{d}_{1}, \cdots, \boldsymbol{d}_{n}, \boldsymbol{d}_{n+1}, \boldsymbol{d}_{n+3}\right), \\
\widehat{\mathbb{D}}^{*} & :=\left(\boldsymbol{d}_{1}^{*}, \cdots, \boldsymbol{d}_{n}^{*}, \boldsymbol{d}_{n+2}^{*}, \boldsymbol{d}_{n+4}^{*}\right)
\end{aligned}
$$

Then $\mathcal{A}$ gives $\left(\right.$ param $\left._{\mathbb{V}}, \widehat{\mathbb{D}}, \widehat{\mathbb{D}}^{*}, \boldsymbol{h}_{b}\right)$ to $\mathcal{B}$, and outputs $b^{\prime}$ if $\mathcal{B}$ outputs $b^{\prime}$. We can see that $\boldsymbol{h}_{0}:=\left(0^{n}, \alpha, 0, \eta, 0,0\right)_{\mathbb{D}}, \boldsymbol{h}_{1}:=$ $\left(0^{n}, \alpha, 0, \eta, 0, \gamma^{\prime}\right)_{\mathbb{D}}$, where $\alpha:=\delta, \eta:=\sigma$ and $\gamma^{\prime}:=\rho$. It is the same as an instance of Problem 2 . If $\mathcal{B}$ succeeds in solving Problem2, so does $\mathcal{A}$ in solving XDLIN problem.
Lemma 4 For all PPT adversary $\mathcal{B}$ for Problem3, there exists a PPT adversary $\mathcal{A}$ such that $\operatorname{Adv}_{\mathcal{B}}^{\mathrm{P} 3}(\lambda) \leq \operatorname{Adv}_{\mathcal{A}}^{\mathrm{P} 1}(\lambda)$.

Proof We can construct a PPT adversary $\mathcal{A}$ for Problem 1 from any PPT adversary $\mathcal{B}$ for Problem $3 . \mathcal{A}$ is given an instance of Problem 1 , (param $\left.1, \mathbb{B}, \widehat{B}^{*}, \boldsymbol{y}_{b}, \kappa G_{1}, \xi G_{2}\right)$. Then $\mathcal{A}$ generates a random linear transformation $W$ on $\mathbb{G}^{n+5}$, and sets

$$
\begin{aligned}
\operatorname{param}_{\mathbb{V}}: & =\operatorname{param}_{\mathrm{P} 1}, \\
\boldsymbol{d}_{i} & :=W\left(0^{i+2}, \kappa G_{1}, 0^{n+2-i}\right) \text { for } i=1, \cdots, n \\
\boldsymbol{d}_{n+1} & :=W\left(\boldsymbol{b}_{1}, 0^{n+2}\right), \boldsymbol{d}_{n+2}:=W\left(0^{n+2}, \kappa G_{1}, 0^{2}\right), \\
\boldsymbol{d}_{n+3} & :=W\left(\boldsymbol{b}_{2}, 0^{n+2}\right), \boldsymbol{d}_{n+4}:=W\left(0^{n+4}, \kappa G_{1}\right), \\
\boldsymbol{d}_{n+5} & :=W\left(\boldsymbol{b}_{3}, 0^{n+2}\right), \\
\boldsymbol{d}_{i}^{*}: & =W\left(0^{i+2}, \xi G_{2}, 0^{n+2-i}\right) \text { for } i=1, \cdots, n \\
\boldsymbol{d}_{n+1}^{*}: & =\left((W)^{-1}\right)^{T}\left(\boldsymbol{b}_{1}^{*}, 0^{n+2}\right), \\
\boldsymbol{d}_{n+2}^{*} & :=\left((W)^{-1}\right)^{T}\left(0^{n+2}, \xi G_{2}, 0^{2}\right), \\
\boldsymbol{d}_{n+3}^{*}: & =\left((W)^{-1}\right)^{T}\left(\boldsymbol{b}_{2}^{*}, 0^{n+2}\right), \\
\boldsymbol{d}_{n+4}^{*} & :=\left((W)^{-1}\right)^{T}\left(0^{n+4}, \xi G_{2}\right), \\
\boldsymbol{d}_{n+5}^{*}: & =\left((W)^{-1}\right)^{T}\left(\boldsymbol{b}_{3}^{*}, 0^{n+2}\right), \\
\boldsymbol{h}_{b} & =W\left(\boldsymbol{y}_{b}, 0^{n+2}\right) \\
\mathbb{D} & :=\left(\boldsymbol{d}_{1}, \cdots, \boldsymbol{d}_{n+5}\right), \mathbb{D}^{*}:=\left(\boldsymbol{d}_{1}^{*}, \cdots, \boldsymbol{d}_{n+5}^{*}\right)
\end{aligned}
$$

We can see that $\left(\mathbb{D}, \mathbb{D}^{*}\right)$ are dual orthonormal bases. $\mathcal{A}$ does not have $\boldsymbol{b}_{2}^{*}$ but it can compute

$$
\begin{aligned}
\widehat{\mathbb{D}} & :=\left(\boldsymbol{d}_{1}, \cdots, \boldsymbol{d}_{n}, \boldsymbol{d}_{n+1}, \boldsymbol{d}_{n+5}\right), \\
\widehat{\mathbb{D}}^{*} & :=\left(\boldsymbol{d}_{1}^{*}, \cdots, \boldsymbol{d}_{n}^{*}, \boldsymbol{d}_{n+2}^{*}, \boldsymbol{d}_{n+4}^{*}\right)
\end{aligned}
$$

Then $\mathcal{A}$ gives $\left(\operatorname{param}_{\mathbb{V}}, \widehat{\mathbb{D}}, \widehat{\mathbb{D}}^{*}, \boldsymbol{h}_{b}\right)$ to $\mathcal{B}$, and outputs $b^{\prime}$ if $\mathcal{B}$ outputs $b^{\prime}$. We can see that $\boldsymbol{h}_{0}:=$ $\left(0^{n}, \alpha, 0, \eta, 0, \gamma^{\prime}\right)_{\mathbb{D}}, \boldsymbol{h}_{1}:=\left(0^{n}, \alpha, 0,0,0, \gamma^{\prime}\right)_{\mathbb{D}}$, where $\alpha:=$ $\delta, \gamma^{\prime}:=\sigma$ and $\eta:=\rho$. It is the same as an instance of Problem 3 . If $\mathcal{B}$ succeeds in solving Problem 3 , so does $\mathcal{A}$ in solving XDLIN problem.

Lemma 5 For all PPT adversary $\mathcal{B}$ for Problem4, there exists a PPT adversary $\mathcal{A}$ such that $\operatorname{Adv}_{\mathcal{B}}^{\mathrm{P} 4}(\lambda) \leq \operatorname{Adv}_{\mathcal{A}}^{\mathrm{P} 1^{\prime}}(\lambda)$.

Proof We can construct a PPT adversary $\mathcal{A}$ for Problem $1^{\prime}$ from any PPT adversary $\mathcal{B}$ for Problem4. $\mathcal{A}$ is given an instance of Problem $1^{\prime}\left(\right.$ param $\left._{1^{\prime}}, \mathbb{B}^{*}, \widehat{\mathbb{B}}, \boldsymbol{y}_{b}^{*}, \kappa G_{1}, \xi G_{2}\right)$. Then $\mathcal{A}$ generates a random linear transformation $W$ on $\mathbb{G}^{n+5}$, and sets 


$$
\begin{aligned}
\operatorname{param}_{\mathbb{V}}: & =\text { param }_{\mathrm{P}^{\prime}} \\
\boldsymbol{d}_{i}: & =W\left(0^{i+2}, \kappa G_{1}, 0^{n+2-i}\right) \text { for } i=1, \cdots, n \\
\boldsymbol{d}_{n+1}: & =W\left(0^{n+1}, \kappa G_{1}, 0^{3}\right), \boldsymbol{d}_{n+2}:=W\left(\boldsymbol{b}_{1}, 0^{n+2}\right), \\
\boldsymbol{d}_{n+3}:= & W\left(\boldsymbol{b}_{2}, 0^{n+2}\right), \boldsymbol{d}_{n+4}:=W\left(\boldsymbol{b}_{3}, 0^{n+2}\right), \\
\boldsymbol{d}_{n+5}:= & \left((W)^{-1}\right)^{T}\left(0^{n+4}, \kappa G_{1}\right), \\
\boldsymbol{d}_{i}^{*}:= & \left((W)^{-1}\right)^{T}\left(0^{i+2}, \xi G_{2}, 0^{n+2-i}\right) \\
& \quad \text { for } i=1, \cdots, n \\
\boldsymbol{d}_{n+1}^{*}:= & \left.(W)^{-1}\right)^{T}\left(0^{n+1}, \xi G_{2}, 0^{3}\right), \\
\boldsymbol{d}_{n+2}^{*}:= & \left((W)^{-1}\right)^{T}\left(\boldsymbol{b}_{1}^{*}, 0^{n+2}\right), \\
\boldsymbol{d}_{n+3}^{*}:= & \left((W)^{-1}\right)^{T}\left(\boldsymbol{b}_{2}^{*}, 0^{n+2}\right), \\
\boldsymbol{d}_{n+4}^{*}:= & \left((W)^{-1}\right)^{T}\left(\boldsymbol{b}_{3}^{*}, 0^{n+2}\right), \\
\boldsymbol{d}_{n+5}^{*}:= & \left((W)^{-1}\right)^{T}\left(0^{n+4}, \xi G_{2}\right), \\
\boldsymbol{h}_{b}^{*}= & \left((W)^{-1}\right)^{T}\left(\boldsymbol{y}_{b}^{*}, 0^{n+2}\right) \\
\mathbb{D}: & \left(\boldsymbol{d}_{1}, \cdots, \boldsymbol{d}_{n+5}\right), \mathbb{D}^{*}:=\left(\boldsymbol{d}_{1}^{*}, \cdots, \boldsymbol{d}_{n+5}^{*}\right)
\end{aligned}
$$

We can see that $\left(\mathbb{D}, \mathbb{D}^{*}\right)$ are dual orthonormal bases. $\mathcal{A}$ does not have $\boldsymbol{b}_{2}$ but it can compute

$$
\begin{aligned}
\widehat{\mathbb{D}} & :=\left(\boldsymbol{d}_{1}, \cdots, \boldsymbol{d}_{n}, \boldsymbol{d}_{n+1}, \boldsymbol{d}_{n+5}\right), \\
\widehat{\mathbb{D}}^{*} & :=\left(\boldsymbol{d}_{1}^{*}, \cdots, \boldsymbol{d}_{n}^{*}, \boldsymbol{d}_{n+2}^{*}, \boldsymbol{d}_{n+4}^{*}\right) .
\end{aligned}
$$

Then $\mathcal{A}$ gives $\left(\right.$ param $\left._{\mathbb{V}}, \widehat{\mathbb{D}}, \widehat{\mathbb{D}}^{*}, \boldsymbol{h}_{b}^{*}\right)$ to $\mathcal{B}$, and outputs $b^{\prime}$ if $\mathcal{B}$ outputs $b^{\prime}$. We can see that $\boldsymbol{h}_{0}^{*}:=\left(0^{n}, 0, \beta, 0, \theta, 0\right)_{\mathbb{D}^{*}}, \boldsymbol{h}_{1}^{*}:=$ $\left(0^{n}, 0, \beta, \tau^{\prime}, \theta, 0\right)_{\mathbb{D}^{*}}$, where $\beta:=\delta, \theta:=\sigma$ and $\tau^{\prime}:=\rho$. It is the same as an instance of Problem4. If $\mathcal{B}$ succeeds in solving Problem4, so does $\mathcal{A}$ in solving XDLIN problem.

Lemma 6 For all $P P T$ adversary $\mathcal{B}$ for Problem5, there exists a PPT adversary $\mathcal{A}$ such that $\operatorname{Adv}_{\mathcal{B}}^{\mathrm{P5}}(\lambda) \leq \operatorname{Adv}_{\mathcal{A}} \mathrm{P}^{\prime}(\lambda)$.

Proof We can construct a PPT adversary $\mathcal{A}$ for Problem1' from any PPT adversary $\mathcal{B}$ for Problem5. $\mathcal{A}$ is given an instance of

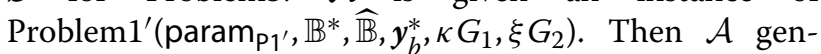
erates a random linear transformation $W$ on $\mathbb{G}^{n+5}$, and sets

$$
\begin{aligned}
\operatorname{param}_{\mathbb{V}}: & =\operatorname{param}_{\mathrm{P}^{\prime}} \\
\boldsymbol{d}_{i}: & =W\left(0^{i+2}, \kappa G_{1}, 0^{n+2-i}\right) \text { for } i=1, \cdots, n \\
\boldsymbol{d}_{n+1}: & =W\left(0^{n+1}, \kappa G_{1}, 0^{3}\right), \boldsymbol{d}_{n+2}:=W\left(\boldsymbol{b}_{1}, 0^{n+2}\right), \\
\boldsymbol{d}_{n+3}:=W\left(\boldsymbol{b}_{3}, 0^{n+2}\right), \boldsymbol{d}_{n+4}:=W\left(\boldsymbol{b}_{2}, 0^{n+2}\right), & \boldsymbol{d}_{n+5}:=W\left(0^{n+4}, \kappa G_{1}\right), \\
\boldsymbol{d}_{i}^{*}:= & \left((W)^{-1}\right)^{T}\left(0^{i+2}, \xi G_{2}, 0^{n+2-i}\right) \\
& \quad \text { for } i=1, \cdots, n
\end{aligned}
$$

$$
\begin{aligned}
\boldsymbol{d}_{n+1}^{*} & :=\left((W)^{-1}\right)^{T}\left(0^{n+1}, \xi G_{2}, 0^{3}\right), \\
\boldsymbol{d}_{n+2}^{*} & :=\left((W)^{-1}\right)^{T}\left(\boldsymbol{b}_{1}^{*}, 0^{n+2}\right), \\
\boldsymbol{d}_{n+3}^{*}: & =\left((W)^{-1}\right)^{T}\left(\boldsymbol{b}_{3}^{*}, 0^{n+2}\right), \\
\boldsymbol{d}_{n+4}^{*} & :=\left((W)^{-1}\right)^{T}\left(\boldsymbol{b}_{2}^{*}, 0^{n+2}\right), \\
\boldsymbol{d}_{n+5}^{*}: & =\left((W)^{-1}\right)^{T}\left(0^{n+4}, \xi G_{2}\right), \\
\boldsymbol{h}_{b}^{*} & =\left((W)^{-1}\right)^{T}\left(\boldsymbol{y}_{b}^{*}, 0^{n+2}\right) \\
\mathbb{D} & :=\left(\boldsymbol{d}_{1}, \cdots, \boldsymbol{d}_{n+5}\right), \mathbb{D}^{*}:=\left(\boldsymbol{d}_{1}^{*}, \cdots, \boldsymbol{d}_{n+5}^{*}\right)
\end{aligned}
$$

We can see that $\left(\mathbb{D}, \mathbb{D}^{*}\right)$ are dual orthonormal bases. $\mathcal{A}$ does not have $\boldsymbol{b}_{2}$ but it can compute

$$
\begin{aligned}
\widehat{\mathbb{D}} & :=\left(\boldsymbol{d}_{1}, \cdots, \boldsymbol{d}_{n}, \boldsymbol{d}_{n+1}, \boldsymbol{d}_{n+5}\right), \\
\widehat{\mathbb{D}}^{*} & :=\left(\boldsymbol{d}_{1}^{*}, \cdots, \boldsymbol{d}_{n}^{*}, \boldsymbol{d}_{n+2}^{*}, \boldsymbol{d}_{n+3}^{*}\right) .
\end{aligned}
$$

Then $\mathcal{A}$ gives $\left(\right.$ param $\left._{\mathbb{V}}, \widehat{\mathbb{D}}, \widehat{\mathbb{D}}^{*}, \boldsymbol{h}_{b}^{*}\right)$ to $\mathcal{B}$, and outputs $b^{\prime}$ if $\mathcal{B}$ outputs $b^{\prime}$. We can see that $\boldsymbol{h}_{0}^{*}:=$ $\left(0^{n}, 0, \beta, \tau^{\prime}, \theta, 0\right)_{\mathbb{D}^{*}}, \boldsymbol{h}_{1}^{*}:=\left(0^{n}, 0, \theta, \tau^{\prime}, 0,0\right)_{\mathbb{D}^{*}}$, where $\beta:=$ $\delta, \tau^{\prime}:=\sigma$ and $\theta:=\rho$. It is the same as an instance of Problem5. If $\mathcal{B}$ succeeds in solving Problem5, so does $\mathcal{A}$ in solving XDLIN problem.

\section{Scheme}

In this section, we present our construction of IPE scheme with simulation-based security.

- IPE.Setup $\left(1^{\lambda}, n\right) \rightarrow(m s k, p p)$ : The setup algorithm selects $\left(\mathbb{B}, \mathbb{B}^{*}\right.$, param $\left._{\mathbb{V}}\right) \stackrel{R}{\leftarrow} \mathcal{G}_{\text {ob }}\left(1^{\lambda}, n+5\right)$, The algorithm outputs $m s k=\left(\widehat{\mathbb{B}}, \widehat{\mathbb{B}}^{*}\right)$, where

$$
\begin{aligned}
\widehat{\mathbb{B}} & =\left\{\boldsymbol{b}_{1}, \cdots, \boldsymbol{b}_{n}, \boldsymbol{b}_{n+1}, \boldsymbol{b}_{n+3}\right\}, \\
\widehat{\mathbb{B}}^{*} & =\left\{\boldsymbol{b}_{1}, \cdots, \boldsymbol{b}_{n}, \boldsymbol{b}_{n+2}, \boldsymbol{b}_{n+4}\right\} \\
\text { and } p p & =\left(1^{\lambda}, \text { param }_{\mathbb{V}}\right)
\end{aligned}
$$

- IPE.Encrypt $(m s k, p p, \boldsymbol{x}) \rightarrow c t_{x}$ : The encryption algorithm samples $\alpha, \eta \in \mathbb{Z}_{q}$ at random and outputs a ciphertext $c t_{x}$ as

$$
c t_{x}=(\boldsymbol{x}, \alpha, 0, \eta, 0,0)_{\mathbb{B}}
$$

- IPE.KeyGen $(m s k, p p, y) \rightarrow s k_{y}$ : The secret key generation algorithm samples $\beta, \theta \in \mathbb{Z}_{q}$ at random and outputs a secret key $s k_{y}$ as

$$
s k_{y}=(y, 0, \beta, 0, \theta, 0)_{\mathbb{B}^{*}}
$$

- IPE.Decryption $\left(p p, c t_{x}, s k_{y}\right) \rightarrow m \in \mathbb{Z}_{p}$ or $\perp$ : The decryption algorithm outputs

$$
d=\tilde{e}\left(c t_{x}, s k_{y}\right)=\tilde{e}\left(G_{1}, G_{2}\right)^{\psi x \cdot y} .
$$

It then attempts to determine $m \in \mathbb{Z}_{q}$ such that $g_{T}^{m}=d$. If there is $m$ that satisfies the equation, the algorithm outputs $m$. Otherwise, it outputs $\perp$. Due to the polynomial-size range of possible values for $m$, the decryption algorithm runs in polynomial time. 
Remark. We stress that the polynomial running time of our decryption algorithm is ensured by restricting the output to lie within a fixed polynomial-size range.

Correctness: For any $c t_{x}$ and $s k_{y}$ in IPE.Encrypt and IPE.KeyGen algorithms respectively, the pairing evaluations in the decryption algorithm compute as follows:

$$
d=\tilde{e}\left(c t_{x}, s k_{y}\right)=\tilde{e}\left(G_{1}, G_{2}\right)^{\psi x \cdot y}=g_{T}^{x \cdot y} .
$$

If the decryption algorithm takes polynomial time in the size of the plaintext space, it will output $m=\boldsymbol{x} \cdot \boldsymbol{y}$ as desired.

\section{Security proof}

In this section, we will prove that our construction is secure under the simulation-based security based on XDLIN assumption in the standard model.

Theorem 1 Under the XDLIN assumption, our proposed scheme is simulation-based secure.

\section{Security proof of our scheme}

In order to finish the security proof, we follow the simulation-based security definition (Caro et al. 2013; O'Neill 2010). A simulator responds to queries by an adversary $\mathcal{A}$ and provides simulated secret keys and simulated ciphertexts to $\mathcal{A}$. The simulator is comprised of three algorithms: $\widetilde{\text { Setup, Encrypt }}$ and KeyGen.

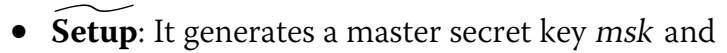
public parameters $p p$, which are transferred to the adversary $\mathcal{A}$. Specially, on input $\left(1^{\lambda}, n\right)$, it sets $(m s k, p p) \leftarrow$ IPE.Setup. The simulator will use the master secret key and the public parameters to respond the queries of $\mathcal{A}$ in Encrypt and $\widetilde{\text { KeyGen }}$

- Encrypt: It simulates the ciphertexts of challenge messages $\boldsymbol{x}^{(1)}, \ldots, \boldsymbol{x}^{\left(q_{1}\right)}$, where $\boldsymbol{x}^{(1)}, \ldots, \boldsymbol{x}^{\left(q_{1}\right)}$ are output by $\mathcal{A}$. $q_{1}$ is the number of the challenge messages. Let $q_{2}$ be the number of secret key queries in the first stage. Encrypt receives as input $m s k, p p$, nonadaptive secret key queries $\boldsymbol{y}^{(1)}, \ldots, \boldsymbol{y}^{\left(q_{2}\right)}$ made by $\mathcal{A}$, together with $\left(\boldsymbol{x}^{(l)} \cdot \boldsymbol{y}^{(1)}, \cdots, \boldsymbol{x}^{(l)} \cdot \boldsymbol{y}^{\left(q_{2}\right)}\right)$ for each $1 \leq l \leq q_{1}$, and the secret keys $\left(\boldsymbol{y}, s k_{y}\right), \cdots,\left(\boldsymbol{y}, s k_{y}\right)$ The normal ciphertext is $(\boldsymbol{x}, \alpha, 0, \eta, 0,0) \mathbb{B}$ generated by IPE.Encrypt, where $\alpha, \eta \stackrel{U}{\leftarrow} \mathbb{Z}_{q}$. The simulated ciphertext is $\left(\boldsymbol{x}, \alpha, 0,0,0, \gamma^{\prime}\right)_{\mathbb{B}}$ generated by Encrypt, where $\gamma^{\prime} \stackrel{\cup}{\leftarrow} \mathbb{Z}_{q}^{\times}$. In order to prove the views of $\mathcal{A}$ in IPE.Encrypt and that in Encrypt have the same distribution, we introduce a new algorithm Encrypt as a transition, where $c t_{x}=\left(\boldsymbol{x}, \alpha, 0, \eta, 0, \gamma^{\prime}\right)_{\mathbb{B}}$.

- KeyGen: It simulates the answer to the second stage queries of $\mathcal{A}$. It receives as input $m s k, p p$, the vector $\boldsymbol{y}$, where $\boldsymbol{y}$ is the secret key query made by $\mathcal{A}$, and the values $\left(\boldsymbol{x}^{(1)} \cdot \boldsymbol{y}\right), \cdots,\left(\boldsymbol{x}^{\left(q_{1}\right)} \cdot \boldsymbol{y}\right)$, where $\boldsymbol{x}^{(1)}, \ldots, \boldsymbol{x}^{\left(q_{1}\right)}$ are the challenge messages. The normal secret key is $(\boldsymbol{y}, 0, \beta, 0, \theta, 0)_{\mathbb{B}^{*}}$ generated by IPE.KeyGen, where $\beta, \theta \stackrel{\cup}{\leftarrow} \mathbb{Z}_{q}$. The simulated secret key is $\left(\boldsymbol{y}, 0, \beta, \tau^{\prime}, 0,0\right)_{\mathbb{B}^{*}}$ generated by KeyGen, where $\tau^{\prime} \stackrel{\cup}{\leftarrow} \mathbb{Z}_{q}^{\times}$. Analogous to Encrypt, we also introduce a new algorithm KeyGen' as a transition, where $s k_{y}=\left(\boldsymbol{y}, 0, \beta, \tau^{\prime}, \theta, 0\right)_{\mathbb{B}^{*}}$.

In our scheme the decryption result should satisfy Eq. (1), so we define the simulated ciphertext and simulated secret key as $\left(\boldsymbol{x}, \alpha, 0,0,0, \gamma^{\prime}\right)_{\mathbb{B}}$ and $\left(\boldsymbol{y}, 0, \beta, \tau^{\prime}, 0,0\right)_{\mathbb{B}^{*}}$, respectively, which would make the Eq. (1) hold as well. Next, we will prove that the output of an ideal world experiment and output of the real world experiment are indistinguishable via a hybrid argument.

Proof A brief overview of the security proof is shown in the Fig. 2. By a standard hybrid argument, we prove the distributions of the outputs in Encrypt and KeyGen are computationally indistinguishable from the normal ciphertexts and the normal secret keys, respectively. We list a series of hybrid experiments $\boldsymbol{H}_{1}, \cdots, \boldsymbol{H}_{6}$ in Table 1 , where $\boldsymbol{H}_{1}$ is the real world experiment and $\boldsymbol{H}_{6}$ is the ideal world experiment. We then prove that hybrid experiment is indistinguishable from the neighboring one.

1 Hybrid $\boldsymbol{H}_{\mathbf{1}}$ : This is the real experiment.

2 Hybrid $\boldsymbol{H}_{2}$ : This experiment is the same as $\boldsymbol{H}_{\mathbf{1}}$ except that the master secret key and the public parameters are generated by $\widetilde{\text { Setup. Namely, the }}$ ciphertext $c t_{x}$ and the secret key $s k_{y}$ are generated by IPE.Encrypt and IPE.KeyGen:

$$
c t_{x}=(\boldsymbol{x}, \alpha, 0, \eta, 0,0)_{\mathbb{B}}, s k_{y}=(\boldsymbol{y}, 0, \beta, 0, \theta, 0)_{\mathbb{B}^{*}} .
$$

3 Hybrid $\boldsymbol{H}_{3}$ : This experiment is the same as $\boldsymbol{H}_{\mathbf{2}}$ except that every challenge ciphertext is $c t_{x}=\left(\boldsymbol{x}, \alpha, 0, \eta, 0, \gamma^{\prime}\right)_{\mathbb{B}}$, which is generated by Encrypt'.

4. $\mathbf{H y b r i d} \boldsymbol{H}_{\mathbf{4}}$ : This experiment is the same as $\boldsymbol{H}_{\mathbf{3}}$ except that every challenge ciphertext is $c t_{\boldsymbol{x}}=\left(\boldsymbol{x}, \alpha, 0,0,0, \gamma^{\prime}\right)_{\mathbb{B}}$, which is generated by Encrypt.

5 Hybrid $\boldsymbol{H}_{\mathbf{5}}$ : This experiment is the same as $\boldsymbol{H}_{\mathbf{4}}$ except that, for every secret key query $\boldsymbol{y}$, the corresponding secret key is $s k_{y}=\left(\boldsymbol{y}, 0, \beta, \tau^{\prime}, \theta, 0\right)_{\mathbb{B}^{*}}$, which is generated by KeyGen'.

6 Hybrid $\boldsymbol{H}_{\mathbf{6}}$ : This experiment is the same as $\boldsymbol{H}_{\mathbf{5}}$ except that, for every secret key query $\boldsymbol{y}$, the corresponding secret key is $s k_{y}=\left(\boldsymbol{y}, 0, \beta, \tau^{\prime}, 0,0\right)_{\mathbb{B}^{*}}$, which is generated by KeyGen. 


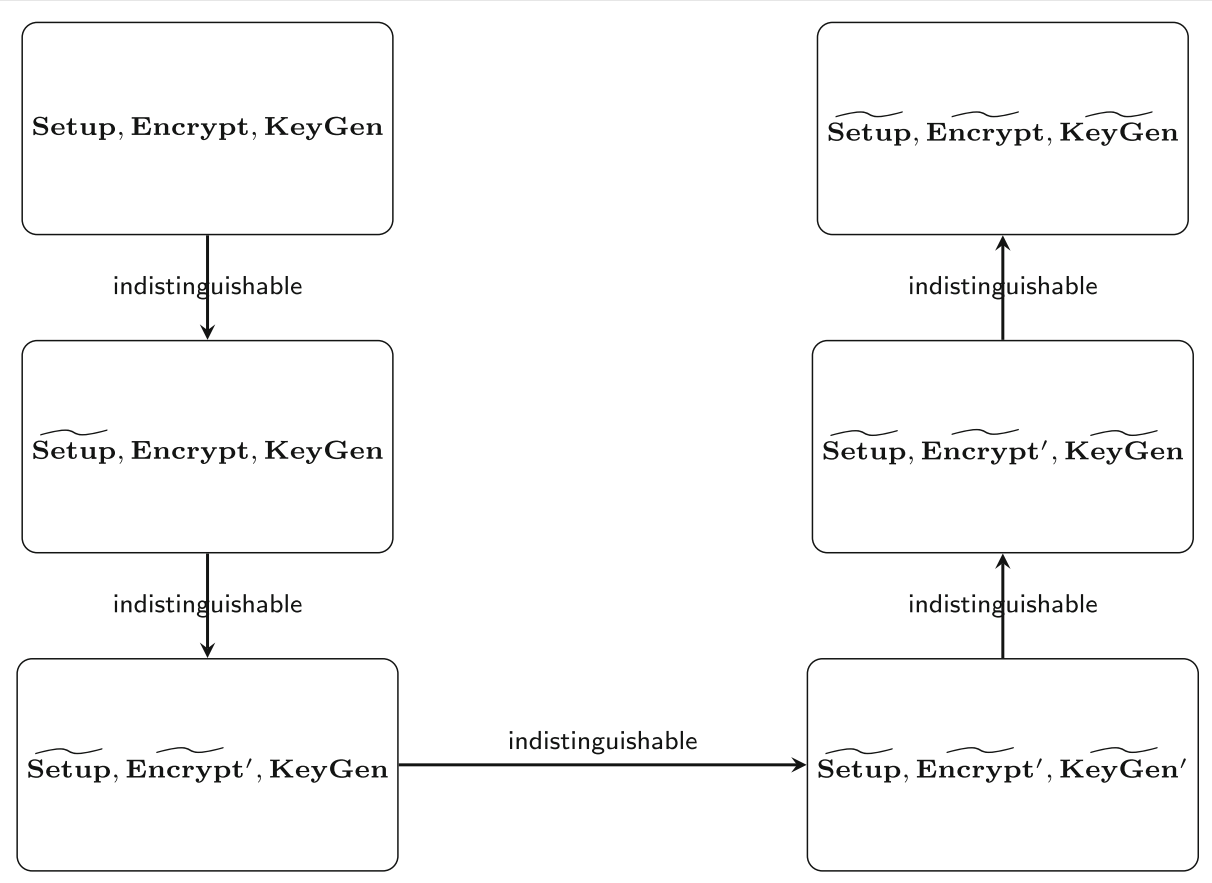

Fig. 2 Framework of Security Proof

Lemma 7 For all PPT adversaries $\mathcal{A}, \boldsymbol{H}_{\mathbf{1}} \stackrel{c}{\approx} \boldsymbol{H}_{\mathbf{2}}$.

Proof Because the master secret key and the public parameters are all generated by IPE.Setup in $\boldsymbol{H}_{\mathbf{1}}$ and $\boldsymbol{H}_{2}$, the view of $\mathcal{A}$ in $\boldsymbol{H}_{1}$ and that in $\boldsymbol{H}_{2}$ has the same distribution.

Lemma 8 Assuming that Problem2 holds, for all PPT adversaries $\mathcal{A}, \mathrm{H}_{2} \stackrel{c}{\approx} \boldsymbol{H}_{3}$.

Proof Suppose that there exists a PPT adversary $\mathcal{A}$ that can distinguish the output distributions of $\boldsymbol{H}_{\mathbf{2}}$ and $\boldsymbol{H}_{3}$. Then, we construct a PPT algorithm $\mathcal{B}$ which is given an instance of Problem $2\left(\right.$ param $\left._{\mathbb{V}}, \widehat{\mathbb{B}}, \widehat{\mathbb{B}}^{*}, \boldsymbol{g}_{b}\right)$ for $b \in\{0,1\}$ and simulates $\boldsymbol{H}_{2}$ and $\boldsymbol{H}_{3}$.

Setup: $\mathcal{B}$ runs IPE.Setup $\left(1^{\lambda}, n\right)$ and outputs $m s k=$ $\left(\widehat{\mathbb{B}}, \widehat{\mathbb{B}}^{*}\right)$ and $p p=\left(1^{\lambda}\right.$, param $\left.\mathbb{V}\right) . \mathcal{B}$ gives $\mathcal{A}$ the public parameters $p p$ and the master secret key msk is only known to $\mathcal{B}$.
Secret Key Queries: To answer the key queries made by $\mathcal{A}, \mathcal{B}$ runs algorithm IPE.KeyGen to respond with $s k_{y}=$ $(\boldsymbol{y}, 0, \beta, 0, \theta, 0)_{\mathbb{B}^{*}}$.

Simulated Ciphertexts: $\mathcal{B}$ randomly chooses $\mu=$ $\left\{1, \cdots, q_{1}\right\}$, where $q_{1}$ is the number of the ciphertext queries asked by adversary $\mathcal{A}$. To answer the ciphertext query that $\mathcal{A}$ makes, $\mathcal{B}$ chooses $\alpha, \eta \stackrel{\cup}{\leftarrow} \mathbb{Z}_{q}$ and $\gamma^{\prime} \stackrel{\cup}{\leftarrow} \mathbb{Z}_{q}^{\times}$ and computes $c t_{x}$ as

$$
\begin{aligned}
c t_{x} & =\sum_{l=1}^{n} x_{l} \boldsymbol{b}_{l}+\alpha \boldsymbol{b}_{n+1}+\eta \boldsymbol{b}_{n+3}+\gamma^{\prime} \boldsymbol{b}_{n+5} \text { if } l<\mu, \\
c t_{x} & =\sum_{l=1}^{n} x_{l} \boldsymbol{b}_{l}+\boldsymbol{g}_{b} \text { if } l=\mu, \\
c t_{x} & =\sum_{i=l}^{n} x_{l} \boldsymbol{b}_{l}+\alpha \boldsymbol{b}_{n+1}+\eta \boldsymbol{b}_{n+3} \text { if } l>\mu .
\end{aligned}
$$

We analyse that the view of $\mathcal{A}$ is composed of the public parameters and the answers of the secret key queries

Table 1 Hybrid argument sequence with structure of ciphertexts and secret keys

\begin{tabular}{lll}
\hline Hybrid argument & Ciphertexts & Secret keys \\
\hline Hybrid $\boldsymbol{H}_{\mathbf{2}}$ & $(\boldsymbol{x}, \alpha, 0, \eta, 0,0)_{\mathbb{B}}$ & $(\boldsymbol{y}, 0, \beta, 0, \theta, 0)_{\mathbb{B}^{*}}$ \\
Hybrid $\boldsymbol{H}_{\mathbf{3}}$ & $\left(\boldsymbol{x}, \alpha, 0, \eta, 0, \gamma^{\prime}\right)_{\mathbb{B}}$ & $(\boldsymbol{y}, 0, \beta, 0, \theta, 0)_{\mathbb{B}^{*}}$ \\
Hybrid $\boldsymbol{H}_{\mathbf{4}}$ & $\left(\boldsymbol{x}, \alpha, 0,0,0, \gamma^{\prime}\right)_{\mathbb{B}}$ & $(\boldsymbol{y}, 0, \beta, 0, \theta, 0)_{\mathbb{B}^{*}}$ \\
Hybrid $\boldsymbol{H}_{\mathbf{5}}$ & $\left(\boldsymbol{x}, \alpha, 0,0,0, \gamma^{\prime}\right)_{\mathbb{B}}$ & $\left(\boldsymbol{y}, 0, \beta, \tau^{\prime}, \theta, 0\right)_{\mathbb{B}^{*}}$ \\
Hybrid $\boldsymbol{H}_{\mathbf{6}}$ & $\left(\boldsymbol{x}, \alpha, 0,0,0, \gamma^{\prime}\right)_{\mathbb{B}}$ & $\left(\boldsymbol{y}, 0, \beta, \boldsymbol{\tau}^{\prime}, 0,0\right)_{\mathbb{B}^{*}}$ \\
\hline
\end{tabular}


and the ciphertext queries. The public parameters in $\boldsymbol{H}_{2}$ and $\boldsymbol{H}_{3}$ are all generated by IPE.Setup and thus have the same distribution, similar to the answers to the secret key queries. It can be seen that if $b=0$, the answer is distributed as in $\boldsymbol{H}_{2}$, if $b=1$, the answer is distributed as in $\mathrm{H}_{3}$.

Lemma 9 Assuming that Problem3 holds, for all PPT adversaries $\mathcal{A}, \boldsymbol{H}_{3} \stackrel{c}{\approx} \boldsymbol{H}_{4}$.

Proof Suppose that there exists a PPT adversary $\mathcal{A}$ that can distinguish the output distributions of $\boldsymbol{H}_{\mathbf{3}}$ and $\boldsymbol{H}_{4}$. Then, we construct a PPT algorithm $\mathcal{B}$ which is given an instance of Problem3 $\left(\right.$ param $\left._{\mathbb{V}}, \widehat{\mathbb{B}}, \widehat{\mathbb{B}}^{*}, \boldsymbol{g}_{b}\right)$ for $b \in\{0,1\}$ and simulates $\boldsymbol{H}_{3}$ and $\boldsymbol{H}_{4}$.

Setup: $\mathcal{B}$ runs IPE.Setup $\left(1^{\lambda}, n\right)$ and outputs $m s k=$ $\left(\widehat{\mathbb{B}}, \widehat{\mathbb{B}}^{*}\right)$ and $p p=\left(1^{\lambda}\right.$, param $\left._{\mathbb{V}}\right)$. $\mathcal{B}$ gives $\mathcal{A}$ the public parameters $p p$ and the master secret key $m s k$ is only known to $\mathcal{B}$.

Secret Key Queries: To answer the key queries made by $\mathcal{A}, \mathcal{B}$ runs algorithm IPE.KeyGen to respond with $s k_{y}=$ $(\boldsymbol{y}, 0, \beta, 0, \theta, 0)_{\mathbb{B}^{*}}$.

Simulated Ciphertexts: $\mathcal{B}$ randomly chooses $\mu=$ $\left\{1, \cdots, q_{1}\right\}$, where $q_{1}$ is the number of the ciphertext queries asked by adversary $\mathcal{A}$. To answer the ciphertext query that $\mathcal{A}$ makes, $\mathcal{B}$ chooses random $\alpha, \eta \stackrel{\cup}{\leftarrow} \mathbb{Z}_{q}$ and $\gamma^{\prime} \stackrel{\cup}{\leftarrow} \mathbb{Z}_{q}^{\times}$and computes and answers as

$$
\begin{aligned}
c t_{x} & =\sum_{l=1}^{n} x_{l} \boldsymbol{b}_{l}+\alpha \boldsymbol{b}_{n+1}+\gamma^{\prime} \boldsymbol{b}_{n+5} \text { if } l<\mu, \\
c t_{x} & =\sum_{l=1}^{n} x_{l} \boldsymbol{b}_{l}+\boldsymbol{g}_{b} \text { if } l=\mu, \\
c t_{x} & =\sum_{l=1}^{n} x_{l} \boldsymbol{b}_{l}+\alpha \boldsymbol{b}_{n+1}+\eta \boldsymbol{b}_{n+3}+\gamma^{\prime} \boldsymbol{b}_{n+5} \text { if } l>\mu .
\end{aligned}
$$

We analyse that the view of $\mathcal{A}$ is composed of the public parameters and the answers of the secret key queries and the ciphertext queries. The public parameters in $\boldsymbol{H}_{3}$ and $\boldsymbol{H}_{\mathbf{4}}$ are all generated by IPE.Setup and thus have the same distribution, similar to the answers to the secret key queries. It can be seen that if $b=0$, the answer is distributed as in $\boldsymbol{H}_{3}$, if $b=1$, the answer is distributed as in $\boldsymbol{H}_{4}$.

Lemma 10 Assuming that Problem4 holds, for all PPT adversaries $\mathcal{A}, \boldsymbol{H}_{\mathbf{4}} \stackrel{c}{\approx} \boldsymbol{H}_{5}$.
Proof Suppose that there exists a PPT adversary $\mathcal{A}$ that can distinguish the output distributions of $\boldsymbol{H}_{\mathbf{4}}$ and $\boldsymbol{H}_{5}$. Then, we construct a PPT algorithm $\mathcal{B}$ which is given an instance of Problem4 $\left(\right.$ param $_{\mathbb{V}}, \widehat{\mathbb{B}}, \widehat{\mathbb{B}}^{*}, \boldsymbol{g}_{b}^{*}$ ) for $b \in\{0,1\}$ and simulates $\boldsymbol{H}_{\mathbf{4}}$ and $\boldsymbol{H}_{5}$.

Setup: $\mathcal{B}$ runs IPE.Setup $\left(1^{\lambda}, n\right)$ and outputs $m s k=$ $\left(\widehat{\mathbb{B}}, \widehat{B}^{*}\right)$ and $p p=\left(1^{\lambda}\right.$, param $\left._{\mathbb{V}}\right) . \mathcal{B}$ gives $\mathcal{A}$ the public parameters $p p$ and the master secret key msk is only known to $\mathcal{B}$.

Ciphertexts Queries: To answer every ciphertext query that $\mathcal{A}$ makes, $\mathcal{B}$ chooses random $\alpha \stackrel{\cup}{\leftarrow} \mathbb{Z}_{q}$ and $\gamma^{\prime} \stackrel{\cup}{\leftarrow} \mathbb{Z}_{q}^{\times}$, runs Encrypt, and answers as $c t_{\boldsymbol{x}}=\left(\boldsymbol{x}, \alpha, 0,0,0, \gamma^{\prime}\right)_{\mathbb{B}}$.

Simulated Secret Keys: $\mathcal{B}$ randomly chooses $v=$ $\left\{1, \cdots, q_{2}\right\}$, where $q_{2}$ is the number of the secret key queries asked by adversary $\mathcal{A}$. To answer the secret key query that $\mathcal{A}$ makes, $\mathcal{B}$ chooses $\beta, \theta \stackrel{\cup}{\leftarrow} \mathbb{Z}_{q}$ and $\tau^{\prime} \stackrel{\cup}{\leftarrow} \mathbb{Z}_{q}^{\times}$ and computes and answers as

$$
\begin{aligned}
& s k_{\boldsymbol{y}}=\sum_{j=1}^{n} y_{j} \boldsymbol{b}_{j}^{*}+\beta \boldsymbol{b}_{n+2}^{*}+\tau^{\prime} \boldsymbol{b}_{n+3}^{*}+\theta \boldsymbol{b}_{n+4}^{*} \text { if } j<v, \\
& s k_{y}=\sum_{j=1}^{n} y_{j} \boldsymbol{b}_{j}^{*}+\boldsymbol{g}_{b} \text { if } j=v, \\
& s k_{y}=\sum_{j=1}^{n} y_{j} \boldsymbol{b}_{j}^{*}+\beta \boldsymbol{b}_{n+2}^{*}+\theta \boldsymbol{b}_{n+4}^{*} \text { if } j>v .
\end{aligned}
$$

We analyse that the view of $\mathcal{A}$ is composed of the public parameters and the answers of the ciphertexts queries and the secret key queries. The public parameters in $\boldsymbol{H}_{\mathbf{4}}$ and $\boldsymbol{H}_{\mathbf{5}}$ are all generated by IPE.Setup and thus have the same distribution, similar to the answers to ciphertexts queries where $c t_{\boldsymbol{x}}$ in $\boldsymbol{H}_{\mathbf{4}}$ and $\boldsymbol{H}_{\mathbf{5}}$ are all generated by Encrypt. It can be seen that if $b=0$, the answer is distributed as in $\boldsymbol{H}_{4}$, if $b=1$, the answer is distributed as in $\boldsymbol{H}_{\mathbf{5}}$.

Lemma 11 Assuming that Problem5 holds, for all PPT adversaries $\mathcal{A}, \boldsymbol{H}_{\mathbf{5}} \stackrel{c}{\approx} \boldsymbol{H}_{6}$.

Proof Suppose that there exists a PPT adversary $\mathcal{A}$ that can distinguish the output distributions of $\boldsymbol{H}_{\mathbf{5}}$ and $\boldsymbol{H}_{\mathbf{6}}$. Then, we construct a PPT algorithm $\mathcal{B}$ which is given an instance of Problem5 $\left(\right.$ param $_{\mathbb{V}}, \widehat{\mathbb{B}}, \widehat{\mathbb{B}}^{*}, \boldsymbol{g}_{b}^{*}$ ) for $b \in\{0,1\}$ and simulates $\boldsymbol{H}_{\mathbf{5}}$ and $\boldsymbol{H}_{\mathbf{6}}$.

Setup: $\mathcal{B}$ runs IPE.Setup $\left(1^{\lambda}, n\right)$ and outputs msk = $\left(\widehat{\mathbb{B}}, \widehat{\mathbb{B}}^{*}\right)$ and $p p=\left(1^{\lambda}\right.$, param $\left._{\mathbb{V}}\right)$. $\mathcal{B}$ gives $\mathcal{A}$ the public parameters $p p$ and the master secret key $m s k$ is only known to $\mathcal{B}$. 
Table 2 Performance comparison of our IPE scheme

\begin{tabular}{lllllll}
\hline & $\begin{array}{l}\text { BJK15(Bishop et al. } \\
\text { 2015) }\end{array}$ & $\begin{array}{l}\text { DDM16(Datta et al. } \\
\text { 2017) }\end{array}$ & $\begin{array}{l}\text { TAO16(Tomida } \\
\text { et al 2016) }\end{array}$ & $\begin{array}{l}\text { ZZL17(Zhao et al. } \\
\text { 2018) }\end{array}$ & $\begin{array}{l}\text { ZZL18(Zhao et al. } \\
\text { 2018) }\end{array}$ & Ours \\
\hline MSK & $\left(8 n^{2}+8\right) \ell_{\mathbb{Z}_{q}}$ & $\left(8 n^{2}+12 n+28\right) \ell_{\mathbb{Z}_{q}}$ & $\left(4 n^{2}+18 n+20\right) \ell_{\mathbb{Z}_{q}}$ & $\left(6 n^{2}+10 n+24\right) \ell_{\mathbb{Z}_{q}}$ & $\left(2 n^{2}+18 n+36\right) \ell_{\mathbb{Z}_{q}}$ & $\left(2 n^{2}+14 n+20\right) \ell_{\mathbb{Z}_{q}}$ \\
CT & $(2 n+2) \ell_{\mathbb{G}_{1}}$ & $(4 n+8) \ell_{\mathbb{G}_{1}}$ & $(2 n+5) \ell_{\mathbb{G}_{1}}$ & $(2 n+4) \ell_{\mathbb{G}_{1}}$ & $(n+6) \ell_{\mathbb{G}_{1}}$ & $(n+5) \ell_{\mathbb{G}_{1}}$ \\
SK & $(2 n+2) \ell_{\mathbb{G}_{2}}$ & $(4 n+8) \ell_{\mathbb{G}_{2}}$ & $(2 n+5) \ell_{\mathbb{G}_{2}}$ & $(2 n+4) \ell_{\mathbb{G}_{2}}$ & $(n+6) \ell_{\mathbb{G}_{2}}$ & $(n+5) \ell_{\mathbb{G}_{2}}$ \\
KeyGen & $2 n+2$ & $4 n+8$ & $2 n+5$ & $2 n+4$ & $n+6$ & $n+5$ \\
Encrypt & $2 n+2$ & $4 n+8$ & $2 n+5$ & $2 n+4$ & $n+6$ & $n+5$ \\
Decrypt & $2 n+2$ & $4 n+8$ & $2 n+5$ & $2 n+4$ & $n+6$ & $n+5$ \\
Assumption & SXDH & SXDH & XDLIN & SXDH & XDLIN & XDLIN \\
Security & IND & IND & IND & SIM & SIM & SIM
\end{tabular}

Legends: $n$ represents dimension of the vectors. All schemes utilize asymmetric bilinear maps over two groups $\mathbb{G}_{1}$ and $\mathbb{G}_{2}$ of order $q . \ell_{\mathbb{G}}$ is the bit length to represent an element in group $\mathbb{G}$

Ciphertexts Queries: To answer every ciphertext query that $\mathcal{A}$ makes, $\mathcal{B}$ chooses random $\alpha \stackrel{\cup}{\leftarrow} \mathbb{Z}_{q}$ and $\gamma^{\prime} \stackrel{\cup}{\leftarrow} \mathbb{Z}_{q}^{\times}$, runs Encrypt, and answers as $c t_{\boldsymbol{x}}=\left(\boldsymbol{x}, \alpha, 0,0,0, \gamma^{\prime}\right)_{\mathbb{B}}$.

Simulated Secret Keys: $\mathcal{B}$ randomly chooses $v=$ $\left\{1, \cdots, q_{2}\right\}$, where $q_{2}$ is the number of the ciphertext queries asked by adversary $\mathcal{A}$. To answer the secret key query that $\mathcal{A}$ makes, $\mathcal{B}$ chooses $\beta, \theta \stackrel{\cup}{\leftarrow} \mathbb{Z}_{q}$ and $\tau^{\prime} \stackrel{\cup}{\leftarrow} \mathbb{Z}_{q}^{\times}$ and computes and answers as

$$
\begin{aligned}
& s k_{y}=\sum_{j=1}^{n} y_{j} \boldsymbol{b}_{j}^{*}+\beta \boldsymbol{b}_{n+2}^{*}+\tau^{\prime} \boldsymbol{b}_{n+3}^{*}+\text { if } j<v, \\
& s k_{y}=\sum_{j=1}^{n} y_{j} \boldsymbol{b}_{j}^{*}+\boldsymbol{g}_{b}^{*} \text { if } j=v, \\
& s k_{y}=\sum_{j=1}^{n} y_{j} \boldsymbol{b}_{j}^{*}+\beta \boldsymbol{b}_{n+2}^{*}+\tau^{\prime} \boldsymbol{b}_{n+3}^{*}+\theta \boldsymbol{b}_{n+4}^{*} \text { if } j>v .
\end{aligned}
$$

We analyse that the view of $\mathcal{A}$ is composed of the public parameters and the answers of the ciphertexts queries and the secret key queries. The public parameters in $\boldsymbol{H}_{\mathbf{5}}$ and $\boldsymbol{H}_{\mathbf{6}}$ are all generated by IPE.Setup and thus have the same distribution, similar to the answers to ciphertexts queries where $c t_{x}$ in $\boldsymbol{H}_{\mathbf{5}}$ and $\boldsymbol{H}_{\mathbf{6}}$ are all generated by Encrypt. It can be seen that if $b=0$, the answer is distributed as in $\boldsymbol{H}_{\mathbf{5}}$, if $b=1$, the answer is distributed as in $\boldsymbol{H}_{\mathbf{6}}$.

So we complete the proof.

\section{Comparison}

To demonstrate the advantage of our IPE scheme, we compare it with some related schemes (Bishop et al. 2015; Tomida et al. 2016; Datta et al. 2017; Zhao et al. 2018; Zhao et al. 2018) in the Table 2. Performance in our scheme is superior to that in the previous schemes in both storage complexity and computation complexity. Our scheme has shorter secret keys and ciphertexts. Additionally, our scheme is secure under weaker assumptions than other schemes. IND and SIM mean indistinguishabilitybased security and simulation-based security, respectively. KeyGen and Encrypt mean scalar multiplication on a cyclic group of IPE.KeyGen algorithm and IPE.Encrypt algorithm, respectively, and Decrypt means pairing operation on a bilinear pairing group of IPE.Decryption algorithm.

\section{Conclusion}

In this paper, we presented an efficient private-key inner product encryption scheme which achieves simulationbased security. Our scheme utilizes asymmetric bilinear pairing groups of prime order under the XDLIN assumption. There are still some open problems for inner product encryption can be explored and researched further. One of the problems is to build unbounded FE schemes for different functionalities, such as Quadratic Polynomials (Baltico et al. 2017). Another one is to construct a Multi-Input inner product encryption scheme under simulation-based security. Abdalla et al. (2017).

Acknowledgements

We would like to thank the anonymous reviewers for detailed comments and useful feedback.

\section{Authors' contributions}

The first author constructed the scheme with careful security proofs and wrote the manuscript. The second author reviewed the manuscript and checked the validity of the scheme and the security proofs. He also proofread the manuscript and corrected the grammar mistakes. The third and the fourth authors joined the discussion of the work and designed the whole figures and tables of the manuscript. All author(s) read and approved the final manuscript.

\section{Funding}

This work is supported by National Natural Science Foundation of China (61872152), the Major Program of Guangdong Basic and Applied Research (2019B030302008), and Science and Technology Program of Guangzhou (201902010081). 


\section{Availability of data and materials}

Not applicable.

\section{Competing interests}

The authors declare that they have no competing interests.

Received: 4 August 2020 Accepted: 15 December 2020

Published online: 06 January 2021

\section{References}

Abdalla M, Bourse F, Caro AD, Pointcheval D (2015) Simple functional encryption schemes for inner products. In: Katz J (ed). Public-Key Cryptography - PKC 2015. Springer, Berlin. pp 733-751

Abdalla M, Bourse F, Caro AD, Pointcheval D (2016) Better security for functional encryption for inner product evaluations. IACR Cryptol ePrint Arch 2016:11

Abdalla M, Gay R, Raykova M, Wee H (2017) Multi-input inner-product functional encryption from pairings. In: Annual International Conference on the Theory and Applications of Cryptographic Techniques. Springer International Publishing, Cham. pp 601-626

Abe M, Nishimaki R, Chase M, David B, Kohlweiss M, Ohkubo M (2016) Constant-size structure-preserving signatures: Generic constructions and simple assumptions. J Cryptol 29(4):833-878

Agrawal S, Agrawal S, Badrinarayanan S, Kumarasubramanian A, Prabhakaran M, Sahai A (2015) On the practical security of inner product functional encryption. Public-Key Cryptography - PKC $2015: 777-798$

Agrawal S, Libert B, Maitra M, Titiu R (2020) Adaptive simulation security for inner product functional encryption. Public-Key Cryptography -PKC 2020 12110:34-64

Agrawal S, Libert B, Stehle D (2016) Fully secure functional encryption for inner products, from standard assumptions. Advances in Cryptology - CRYPTO $2016: 333-362$

Attrapadung N, Libert B (2010) Functional encryption for inner product: Achieving constant-size ciphertexts with adaptive security or support for negation. In: Nguyen PQ, Pointcheval D (eds). Public Key Cryptography PKC 2010. Springer Berlin Heidelberg, Berlin. pp 384-402

Baltico CEZ, Catalano D, Fiore D, Gay R (2017) Practical functional encryption for quadratic functions with applications to predicate encryption. Advances in Cryptology - CRYPTO 2017:67-98

Bishop A, Jain A, Kowalczyk L (2015) Function-hiding inner product encryption. In: Advances in Cryptology - ASIACRYPT 2015. Springer Berlin Heidelberg, Berlin. Vol. 9452. pp 470-491

Boneh D, Franklin M (2001) Identity-based encryption from the weil pairing. Springer Berlin Heidelberg, Berlin. Vol. 32. pp 213-229

Caro AD, lovino V, Jain A, O'Neill A, Paneth O, Persiano G (2013) On the achievability of simulation-based security for functional encryption. In: Canetti R, Garay JA (eds). Advances in Cryptology - CRYPTO 2013. Springer Berlin Heidelberg, Berlin. pp 519-535

Caro AD, lovino V, Persiano G (2012) Fully secure hidden vector encryption. In: Pairing-Based Cryptography - Paring 2012. Springer Berlin Heidelberg, Berlin. pp 102-121

Dan B, Sahai A, Waters B (2011) Functional encryption: Definitions and challenges. In: Theory of Cryptography Conference. Springer Berlin Heidelberg, Berlin. pp 253-273

Datta P, Dutta R, Mukhopadhyay S (2016) Functional encryption for inner product with full function privacy. Public-Key Cryptography - PKC 2016:164-195

Datta P, Dutta R, Mukhopadhyay S (2017) Strongly full-hiding inner product encryption. Theor Comput Sci 667:16-50

Datta P, Okamoto T, Tomida J (2018) Full-hiding (unbounded) multi-input inner product functional encryption from the K-linear assumption. In: Public-Key Cryptography, PKC 2018. Springer International Publishing, Cham Vol. 10770. pp 245-277

Dufour-Sans E, Pointcheval D (2019) Unbounded Inner-Product Functional Encryption with Succinct Keys. In: Deng RH, Gauthier-Umaña V, Ochoa M, Yung M (eds). Applied Cryptography and Network Security. Springer International Publishing, Cham. pp 426-441

Garg S, Gentry C, Halevi S, Raykova M, Sahai A, Waters B (2016) Candidate indistinguishability obfuscation and functional encryption for all circuits. SIAM J Comput 45:882-929
Goldwasser S, Gordon SD, Goyal V, Jain A, Katz J, Liu F-H, Sahai A, Shi E, Zhou H-S (2014) Multi-input functional encryption. In: Nguyen PQ, Oswald E (eds). Advances in Cryptology - EUROCRYPT 2014. Springer Berlin Heidelberg, Berlin. pp 578-602

Goyal V, Pandey O, Sahai A, Waters B (2006) Attribute-based encryption for fine-grained access control of encrypted data. In: Extended abstract to appear in ACM CCS 2006 Vol. 89-98. pp 89-98

lovino V, Persiano G (2008) Hidden-vector encryption with groups of prime order. In: Pairing-Based Cryptography - Paring 2008. Springer Berlin Heidelberg, Berlin. Vol. 5209. pp 75-88

Katz J, Sahai A, Waters B (2008) Predicate encryption supporting disjunctions, polynomial equations, and inner products. In: Smart N (ed). Advances in Cryptology - EUROCRYPT 2008. Springer, Berlin. pp 146-162

Kawai Y, Takashima K (2013) Predicate- and attribute-hiding inner product encryption in a public key setting. Lect Notes Comput Sci 8365:113-130

Kim S, Kim J, Seo J (2019) A new approach to practical function-private inner product encryption. Theoretical Computer Science 783:22-40

Kim S, Lewi K, Mandal A, Montgomery H, Wu DJ (2018) Function-hiding inner product encryption is practical. In: Catalano D, De Prisco R (eds). Security and Cryptography for Networks. Springer International Publishing, Cham. pp 544-562

Lewko A, Okamoto T, Sahai A, Takashima K, Waters B (2010) Fully secure functional encryption: Attribute-based encryption and (hierarchical) inner product encryption. In: Gilbert H (ed). Advances in Cryptology EUROCRYPT 2010. Springer, Berlin. pp 62-91

Okamoto T (2011) Achieving short ciphertexts or short secret-keys for adaptively secure general inner product encryption. Cans 77(2-3):725-771

Okamoto T, Takashima K (2009) Hierarchical predicate encryption for inner-products. In: International Conference on Advances in Cryptology-Asiacrypt. Springer Berlin Heidelberg, Berlin. Vol. 5912. pp 214-231

Okamoto T, Takashima K (2012) Adaptively attribute-hiding (hierarchical) inner product encryption. In: Pointcheval D, Johansson T (eds). Advances in Cryptology - EUROCRYPT 2012. Springer Berlin Heidelberg, Berlin. pp 591-608

Okamoto T, Takashima K (2012) Fully secure unbounded inner-product and attribute-based encryption. In: Wang X, Sako K (eds). Advances in Cryptology - ASIACRYPT 2012. Springer Berlin Heidelberg, Berlin pp 349-366

Okamoto T, Takashima K (2015) Dual pairing vector spaces and their applications. IEICE Trans Fundam Electron Commun Comput Sci E98.A(1):3-15

O'Neill A (2010) Definitional issues in functional encryption. IACR Cryptol ePrint Arch 2010:556

Park JH (2011) Inner-product encryption under standard assumptions. Des Codes Crypt 58:235-257

Shen E, Shi E, Waters B (2009) Predicate privacy in encryption systems. In: Theory of Cryptography. Springer Berlin Heidelberg, Berlin. pp 457-473

Tomida J, Abe M, Okamoto T (2016) Efficient functional encryption for inner-product values with full-hiding security. Information Security 9866:408-425

Tomida J, Takashima K (2018) Unbounded Inner Product Functional Encryption from Bilinear Maps. In: Peyrin T, Galbraith S (eds). Advances in Cryptology ASIACRYPT 2018. Springer International Publishing, Cham. pp 609-639

Zhang Y, Li Y, Wang Y (2019) Efficient inner encryption for mobile clients with constrained computation capacity. Int J Innov Comput Inf Control 15(1):209-226

Zhao Q, Zeng Q, Liu X (2018) Improved construction for inner product functional encryption. Secur Commu Netw 2018:1-12

Zhao Q, Zeng Q, Liu X, Xu H (2018) Simulation-based security of function-hiding inner product encryption. Sci Chin Inf Sc 61 (4):048102

Zhenlin T, Wei Z (2015) A predicate encryption scheme supporting multiparty cloud computation. In: 2015 International Conference on Intelligent Networking and Collaborative Systems. pp 252-256

\section{Publisher's Note}

Springer Nature remains neutral with regard to jurisdictional claims in published maps and institutional affiliations. 